\title{
DISCLAIMER
}

This report was prepared as an account of work sponsored by an agency of the United States Government. Neither the United States Government nor any agency thereof, nor any of their employees, makes any warranty, express or implied, or assumes any legal liability or responsibility for the accuracy, complcteness, or usefulness of any information, apparatus, product, or process disclosed, or represents that its use would not infringe privately owned rights. Reference herein to any specific commercial product, process, or service by trade name, trademark, manufacturer, or otherwise does not necessarily constitute or imply its endorsement, recommendation, or favoring by the United States Government or any agency thereof. The views and opinions of authors expressed herein do not necessarily state or reflect those of the United States Government or any agency thereof.

\section{Analysis of Meteorological Data from Three Sites in the Northeastern United States for Application of EPA AIRDOS Code}

\author{
Frank Couveia
}

August 1990 


\section{Table of Contents}

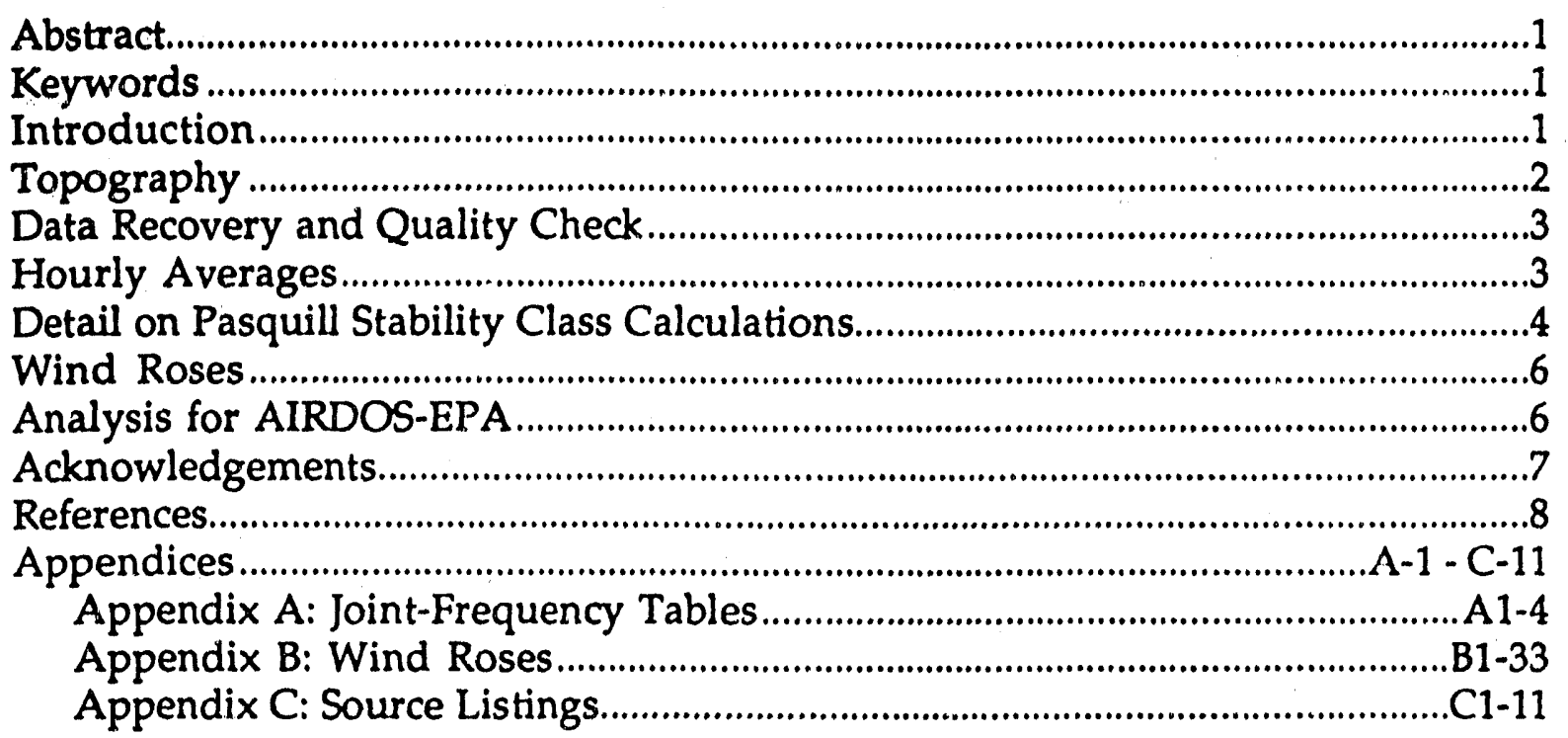




\title{
Analysis of Meteorological Data from \\ Three Sites in the Northeastern United States for Applications of EPA AIRDOS Code
}

\author{
Frank Gouveia
}

\begin{abstract}
Absiract
Meteorological data for the entire year of 1989 were collected at three sites that comprise the Knolls Atomic Power Laboratory: Knolls site, Kesselring site, and Windsor site. These data are 15-min averages of wind speed, wind direction, standard deviation of wind direction, and temperature. Hourly averages were computed and used for all analysis. The Pasquill stability category was computed using procedures established by the Envircnmental Protection Agency. Joint frequency of wind speed, direction, and stability were computed and displayed as wind roses for each stability. The joint frequency tables are presented in the form that is appropriate as meteorological input for the AIRDOS-EPA dispersion model.
\end{abstract}

Keywords

Climatology, meteorological data, dispersion data, KAPL, stability class, jointfrequency diagrams, AIRDOS-EPA model.

\section{Introduction}

Fifteen-minute-average data were collected at three sites for the 1989 calendar year. The three sites are

Knolls site, 2 miles east of Schenectady, New York, Kesselring site, 17 miles north of Schenectudy, New York, and Windsor site, in the town of Windsor, Connecticut.

These sites comprise the Knolls Atomic Power Laboratory (KAPL). Table 1 shows the locations and elevations of the three meteorological towers.

The data were collected using HANDAR 540 data-acquisition systems (DAS). Table 2 is a list of instruments used and their specifications. The instruments were placed at $20 \mathrm{~m}$ above ground level at Knolls, 10 and $43 \mathrm{~m}$ at Kesselring, and $30 \mathrm{~m}$ at Windsor. The $10-\mathrm{m}$ level at Kesselring is well below the tree canopy and, therefore, should not be used for dispersion statistics. The analysis of the 10-m data is included in this paper for comparison with previous data from this level. 
Table 1 -- Locations of the three KAPL sites.

Site Longitude Latitude Elevation (m)

\begin{tabular}{lllr}
\hline Knolls & $73^{\circ} 52^{\prime} 00^{\prime \prime} \mathrm{W}$ & $42^{\circ} 49^{\prime} 24^{\prime \prime} \mathrm{N}$ & 103 \\
Kesselring & $73^{\circ} 56^{\prime} 54^{\prime \prime} \mathrm{W}$ & $43^{\circ} 02^{\prime} 27^{\prime \prime} \mathrm{N}$ & 143 \\
Windsor & $72^{\circ} 42^{\prime} 44^{\prime \prime} \mathrm{W}$ & $41^{\circ} 53^{\prime} 16^{\prime \prime} \mathrm{N}$ & 55
\end{tabular}

Table 2 - Instruments used at KAPL towers.

\begin{tabular}{|c|c|c|c|c|}
\hline Parameter & Instrument & Threshold & Accuracy & Other \\
\hline Wind Speed & $\begin{array}{l}\text { Three-cup } \\
\text { Anemometer }\end{array}$ & $0.5 \mathrm{~m} / \mathrm{s}$ & $\pm 0.15 \mathrm{~m} / \mathrm{s}$ & \\
\hline Wind Direction & Wind Vane & $0.5 \mathrm{~m} / \mathrm{s}$ & $\pm 0.3^{\circ}$ Dista & $\begin{array}{c}\text { constant } \\
4.5 \mathrm{~m}\end{array}$ \\
\hline Temperature & Thermistors & - & $\pm 0.1^{\circ} \mathrm{C}$ & \\
\hline
\end{tabular}

The instruments are polled once every second and averages are computed at 15-min intervals. The standard deviation of horizontal wind direction (sigma theta, $\left.\sigma_{\theta}\right)$ is computed every $15 \mathrm{~min}$ also.

\section{Topography}

The Knolls site ( 2 miles east of Schenectady) is at the southeastern end of the Mohawk River Valley, and near the northern end of the Hudson River Valley, approximately 150 miles from the Atlantic coastline. The Kesselring site is approximately 17 miles north of the Knolls site. The Windsor site is located in the town of Windsor, Connecticut, and is near the middle of the Connecticut River Valley, aproximately 5 miles north of the City of Hartford and 50 miles north of Long Island Sound. The general topography at all three sites is low rolling hills with grassy fields and forests of evergreen and deciduous trees. The towers are in clearings containing one- and two-story buildings. The Windsor tower is on the roof of a building. The Hudson and Connecticut Rivers flow from the north to south. The Mohawk River flows from northwest to southeast. 


\section{Data Recovery and Quality Check}

KAPL personnel transferred the data to IBM-PC compatible ASCII files. Data recovery at the three sites was excellent. From one year of data collection at all three sites, just 1548 15-min averages are missing out of a total possible of 105,120 (1.5\% missing). Most of the missing data (1440 data points) comes from the Knolls site, where the data for first 15 days of November are missing.

During the process of checking the quality of data, it was discovered that $\sigma_{\theta}$ values from Windsor were far too large. The cause of this problem was determined to be the improper calculation of $\sigma_{\theta}$ by the data-acqusition program in the HANDAR 540 DAS. The Windsor DAS contains version 6.1 of the acquisition program. This version is known to calculate $\sigma_{\theta}$ improperly. The DAS at Knolls also has version 6.1 of the program, but the $\sigma_{\theta}$ data do not seem to be as severely affected as the Windsor data. The Kesselring DAS was hit by lightning in August of 1988 , so the DAS was replaced with one containing program version 6.9 . This improved DAS program calculates $\sigma \theta$ using an algorithm suggested by Yamartino (1984). Turner (1986) states that the method of estimation of $\sigma_{\theta}$ by Yamartino gives excellent results over the entire range of possible standard deviations $0^{\circ}$ to $103.9^{\circ}$.

It was decided that the ${ }^{\sigma} \theta$ data from Windsor site would not be used to calculate Pasquill stability class. The wind speed and direction data appear to be unaffected, so wind roses and joint frequency of occurrence of wind speed and direction were computed over all stability classes. Although at the Knolls site we used the same DAS program version as at the Windsor site, the problem with $\sigma_{\theta}$ was not as severe. The Knolls $\sigma_{\theta}$ data will be used to calculate Pasquill stability class.

\section{Hourly Averages}

Hourly averages were computed by the program HOURK (see appendix for source-code listing). This program was written following guidelines published by the U.S. Environmental Protection Agency (EPA) in EPA (1987). Wind speed and temperature were scalar averaged (equation 6.1.1 and 6.2.2 in EPA 1987). Wind direction was weighted with the wind speed and polar averaged (equation 6.1.14). The hourly value of $\sigma_{\theta}$ was computed by the root-mean-squared method (equation 6.1.10).

The wind direction and $\sigma_{\theta}$ should be disregarded during periods when the wind is less than the threshold speed of the anemometer (wind speed $<0.5 \mathrm{~m} / \mathrm{s}$ ). EPA (1987) recommends that the wind direction and $\sigma_{\theta}$ be taken from the latest non-calm measurement. This guideline was implemented while calculating the hourly averages. 


\section{Detail on Pasquill Stability Class Calculations}

The hourly values of wind speed and $\sigma_{\theta}$ were entered into the subroutine SIGCATK (see appendix for listing), which calculates Pasquill stability class A through $G$ according to EPA (1987). The method presented by EPA (1987) was changed to separate class $F$ into classes $F$ and $G$. The algorithm is often called the Modified Sigma Theta (MST) method and is based on an initial estimate of stability modified by time of day and wind speed (Tables 3 and 4). Unstable classes A through D-Day only occur during the daytime and the stable classes D-Night through $G$ only occur during the night. Table 4 shows the modification of the initial estimate according to wind speed. The higher wind speeds force stability towards neutral. Table 3 gives the lower bounds of $\sigma_{\theta}$ classes to determine the initial estimate of Pasquill stability category.

Night refers to the period from one hour before sunset to one hour after sunrise. Sunrise and sunset times are calculated by,

$$
t=\cos ^{-1}\left(-\frac{\sin \phi \sin \delta}{\cos \phi \cos \delta}\right)
$$

where $t$ is the time of day, $\phi$ is the latitude and $\delta$ is the declination of the sun. The sunrise and sunset times are adjusted for the equation of time and longitude.

Table 3 -- Lateral wind direction turbulence criteria for initial estimate of Pasquill stability category. Based on surface roughness of $40 \mathrm{~cm}$ and various instrument heights. Use with Table 4.

Lower bound of standard deviaticn of horizontal wind direction fluctuations, ${ }^{\sigma} \theta$, in degrees

Initial estimate of Pasquill stability category
A
B
C
D
E
F

Instrument height in meters

10

20

30

43

27.4

21.3

15.2

9.1

4.6

2.6
26.3

19.2

13.5

7.8

3.6

1.9
25.6

18.1

12.6

7.1

3.0

1.6
25.1

17.1

11.9

6.5

2.7

1.4 
Table 4 -. Wind speed adjustments for determining final estimate of Pasquill stability category from ${ }^{\sigma} \theta$. Use with Table 3 .

\begin{tabular}{|c|c|c|c|}
\hline & $\begin{array}{l}\text { Initial estimated } \\
\text { category }\end{array}$ & $\begin{array}{l}\text { 10-m scalar wind } \\
\text { speed (WS) }(\mathrm{m} / \mathrm{s})\end{array}$ & $\begin{array}{l}\text { Final estimate of } \\
\text { stability category }\end{array}$ \\
\hline \multirow[t]{4}{*}{ Daytime } & $\bar{A}$ & $\begin{aligned} & W S<3 \\
3< & =W S<4 \\
4< & =W S<6 \\
6< & =W S\end{aligned}$ & $\begin{array}{l}A \\
B \\
C \\
D\end{array}$ \\
\hline & B & $\begin{aligned} & W S<4 \\
4<= & W S<6 \\
6<= & =W S\end{aligned}$ & $\begin{array}{l}\text { B } \\
\text { C } \\
\text { D }\end{array}$ \\
\hline & C & $\begin{aligned} & W S<6 \\
6<= & W S\end{aligned}$ & $\begin{array}{l}C \\
D\end{array}$ \\
\hline & $D, E, F$ or $G$ & ANY & D \\
\hline \multirow[t]{6}{*}{ Nighttime } & A & $\begin{aligned} \text { WS }<2.4 \\
2.4<=\text { WS }<2.9 \\
2.9<=\text { WS }<3.6 \\
3.6<=\text { WS } \\
\text { WS }<2.4 \\
2.4<=\text { WS }<3.0 \\
3.0<=\text { WS }\end{aligned}$ & $\begin{array}{l}G \\
F \\
E \\
D \\
F \\
E \\
D\end{array}$ \\
\hline & c & $\begin{aligned} & W S<2.4 \\
2.4<= & W S\end{aligned}$ & $\begin{array}{l}E \\
D\end{array}$ \\
\hline & $D$ & ANY & D \\
\hline & E & $\begin{aligned} & W S<5.0 \\
& 5.0<=W S\end{aligned}$ & $\begin{array}{l}E \\
D\end{array}$ \\
\hline & F & $\begin{aligned} & W S<3.0 \\
3.0<= & W S<5.0 \\
5.0< & =W S\end{aligned}$ & $\begin{array}{l}E \\
E \\
D\end{array}$ \\
\hline & G & $\begin{aligned} & W S<3.0 \\
3.0<= & W S<5.0 \\
5.0<=W S & =5\end{aligned}$ & $\begin{array}{l}\text { G } \\
\text { E } \\
D\end{array}$ \\
\hline
\end{tabular}




\section{Wind Roses}

Joint frequency of occurence of wind speed and direction for all 7 stability classes were constructed. The neutral stability classes of D-Day and D-Night were combined into one neutral class. Wind direction was separated into 16 classes of $22.5^{\circ}$, centered on the compass direction. Following the meteorological convention, the labeled wind direction is the direction the wind is coming from. Wind speed was separated into 5 classes according to Table 5.

The joint-frequency tables are graphically displayed as wind roses in Appendix B. There are 8 wind roses for each site, one for each of the seven stability classes (A through G) and one for all stabilities together.

\section{Analysis for AIRDOS-EPA}

AIRDOS-EPA (AIR DOSe) is a computer model for annual radiological dose assessment (EPA 1989). EPA has mandated that AIRDOS-EPA be used to calculate offsite dose from all emissions. Summaries of meteorological data are necessary input data for this model. There are four major meteorological inputs required for the AIRDOS-EPA model. These are mixing depth, the frequency of wind directions, the frequency of each Pasquill stability class for each wind direction, and the average wind speeds in meters per second.

Mixing depth is sometimes called atmospheric lid or mixing height. This is the distance from the ground to the top of a layer of vigorous vertical mixing. The value is site specific and changes through the day. It can be estimated from the contour maps in Holtzworth (1972). On a warm, summer day, mixing depth rnay be as high as $4,000 \mathrm{~m}$. At night, the mixing depth may be lower than $200 \mathrm{~m}$. Although it changes with time of day, mixing depth does not affect pollutant concentrations until the plume is many kilometers downwind. It is prudent to choose a mixing depth that is lower than the average daily depth.

\section{Joint-Frequency Tables}

Table 5 -- Wind speed classifications used to construct the wind roses in Appendix B

Class name Wind speed (WS) $(\mathrm{m} / \mathrm{s})$

\begin{tabular}{ll}
\hline Calm & WS $<=1.0$ \\
Weak & $1.0<$ WS $<=3.0$ \\
Moderate & $3.0<$ WS $<=5.0$ \\
Strong & $5.0<$ WS $<=7.0$ \\
Very Strong & $7.0<$ WS
\end{tabular}


The AIRDOS-EPA model requires that wind directions be defined as blowing TOWARD the 16 compass directions. This convention is opposite to that of the joint-frequency tables. The frequency of each wind direction class must be determined as well as the frequency of wind direction class over all eight stabilities. The sum of the frequencies for categories $A$ through $G$ will be equal to 1 for each of the 16 wind directions.

Average wind speed is determined for each wind direction and stability. Reciprocal-averaged wind speeds are also determined. These values are calculated by averaging reciprocals of wind speeds and then inverting the reciprocal averages. The AIRDOS-EPA manual (EPA 1989) warns that all values of average and reciprocal-averaged wind speed for each direction and stability used for calculations in the model must be greater than zero or AIRDOS-EPA will stop with a divisionby-zero error.

The appendix has summaries of the data files necessary for the meteorological input for AIRDOS-EPA for the three KAPL sites. Also, the same data are displayed in the appendix with the columns and rows appropriately labeled.

\section{Acknowledgments}

The author wishes to thank Chuck Foelix of General Electric, Ron Baskett of G-Division at LLNL, and Joseph Shinn of Environmental Sciences Division at LLNL for their valuable help. Work performed under the auspices of the U.S. Department of Energy by Lawrence Livermore National Laboratory under Contract W-7405-ENG-48. 


\section{REFERENCES}

Environmental Protection Agency, 1987, On-Site Meteorological Program Guidance for Regulatory Modeling Applications. EPA-450/4-87-013. U.S. Environmental Protection Agency, Research Triangle Park, NC.

Environmental Protection Agency, 1989, AIRDOS-PC User's Guide and Program Manual. EPA-520/6-89-035. U.S. Environmental Protection Agency, Research Triangle Park, NC.

Holtzworth, G.C., 1972, Mixing Heights. Wind Speeds, and Potential for Urban Air Pollution Throughout the Contiguous United States. U.S. Environmental Protection Agency, Research Triangle Park, NC. Publication Number AP-101.

Turner, D.B., 1986. "Comparison of Three Methods for Calculating the Standard Deviation of the Wind Direction," J. Climate Appl. Meteor., 25, 703-707.

Yamartino, R.J., 1984. "A Comparison of Several "Single-Pass" Estimators of the Standard Deviation of Wind Direction," J. Climate Appl. Meteor., 23, 13621366. 
APPENDIX A

JOINT-FREQUENCY TABLES FOR INPUT TO AIRDOS-EPA PROGRAM 
EREQUENCY TABLES FOR 1989 ALL HOURS, 8399 DATA POINTS KNOLLS SITE FREQUENCY OF EACH STABILITY CLASS FOR EACH WIND DIRECTION WIND TOWARD

FRACTION OF TIME IN EACH STABILITY CLASS

\begin{tabular}{lcccccccc} 
& $\mathbf{A}$ & $\mathbf{B}$ & $\mathbf{C}$ & $\mathbf{D}$ & $\mathbf{E}$ & $\mathbf{F}$ & $\mathbf{G}$ & ALL \\
N & 0.0226 & 0.0293 & 0.1618 & 0.6125 & 0.1332 & 0.0158 & 0.0248 & 0.1582 \\
NNW & 0.0397 & 0.0269 & 0.2103 & 0.5603 & 0.1103 & 0.0167 & 0.0359 & 0.0929 \\
NW & 0.1564 & 0.1401 & 0.3550 & 0.1792 & 0.0912 & 0.0326 & 0.0456 & 0.0366 \\
WNW & 0.2541 & 0.1676 & 0.2595 & 0.1351 & 0.1027 & 0.0378 & 0.0432 & 0.0220 \\
W & 0.2929 & 0.1500 & 0.2071 & 0.1000 & 0.1429 & 0.0429 & 0.0643 & 0.0167 \\
WSW & 0.2214 & 0.1357 & 0.2000 & 0.1286 & 0.1786 & 0.0643 & 0.0714 & 0.0167 \\
SW & 0.1520 & 0.1094 & 0.2584 & 0.2462 & 0.1398 & 0.0456 & 0.0486 & 0.0392 \\
SSW & 0.0893 & 0.0470 & 0.2038 & 0.3793 & 0.2006 & 0.0455 & 0.0345 & 0.0760 \\
S & 0.1161 & 0.0580 & 0.1071 & 0.3728 & 0.1920 & 0.0603 & 0.0938 & 0.0533 \\
SSE & 0.1129 & 0.0538 & 0.1478 & 0.4005 & 0.1452 & 0.0618 & 0.0780 & 0.0443 \\
SE & 0.0815 & 0.0599 & 0.2030 & 0.4243 & 0.1564 & 0.0483 & 0.0266 & 0.0716 \\
ESE & 0.0287 & 0.0271 & 0.2670 & 0.5653 & 0.0801 & 0.0138 & 0.0180 & 0.2243 \\
E & 0.0549 & 0.0248 & 0.1788 & 0.5381 & 0.0991 & 0.0389 & 0.0655 & 0.0673 \\
ENE & 0.0460 & 0.0460 & 0.1632 & 0.3556 & 0.2176 & 0.0628 & 0.1088 & 0.0285 \\
NE & 0.0846 & 0.0498 & 0.1393 & 0.3582 & 0.1741 & 0.0896 & 0.1045 & 0.0239 \\
NNE & 0.0664 & 0.0373 & 0.1245 & 0.3817 & 0.2282 & 0.0539 & 0.1079 & 0.0287 \\
\hline
\end{tabular}

RECIPROCAL-AVERAGED WIND SPEEDS

WIND TOWARD

$\begin{array}{lcccccccc} & \text { A } & \text { B } & C & D & E & \text { F } & \text { G } \\ \text { N } & 1.490 & 1.698 & 2.733 & 2.351 & 1.394 & 1.120 & 1.124 \\ \text { NNW } & 1.474 & 1.601 & 2.422 & 2.006 & 1.231 & 1.112 & 1.070 \\ \text { NW } & 1.291 & 1.564 & 1.730 & 1.480 & 1.096 & 1.298 & 1.138 \\ \text { WNW } & 1.329 & 1.420 & 1.451 & 1.399 & 1.200 & 1.057 & 1.011 \\ \text { W } & 1.227 & 1.235 & 1.326 & 1.222 & 1.094 & 1.062 & 1.091 \\ \text { WSW } & 1.237 & 1.304 & 1.556 & 1.177 & 1.234 & 1.037 & 1.120 \\ \text { SW } & 1.327 & 1.511 & 2.089 & 2.324 & 1.313 & 1.216 & 1.046 \\ \text { SSW } & 1.427 & 1.704 & 2.594 & 2.760 & 1.414 & 1.153 & 1.168 \\ \text { S } & 1.357 & 1.499 & 1.721 & 1.648 & 1.403 & 1.440 & 1.220 \\ \text { SSE } & 1.682 & 1.908 & 2.651 & 1.736 & 1.345 & 1.173 & 1.136 \\ \text { SE } & 1.575 & 2.300 & 3.404 & 2.746 & 1.638 & 1.373 & 1.230 \\ \text { ESE } & 1.783 & 2.691 & 4.098 & 3.876 & 1.783 & 1.400 & 1.231 \\ \text { E } & 1.545 & 2.252 & 3.897 & 2.430 & 1.537 & 1.332 & 1.145 \\ \text { ENE } & 1.839 & 1.806 & 3.468 & 1.931 & 1.276 & 1.346 & 1.133 \\ \text { NE } & 1.851 & 1.742 & 2.433 & 1.602 & 1.254 & 1.230 & 1.108 \\ \text { NNE } & 1.510 & 1.775 & 2.183 & 1.527 & 1.357 & 1.086 & 1.060\end{array}$

\begin{tabular}{ccccccccc} 
NNE & 1.510 & 1.775 & 2.183 & 1.527 & 1.357 & 1.086 & 1.060 \\
WIND TOWARD & & \multicolumn{5}{c}{ AVERAGE WIND SPEEDS } \\
& A & BND SPEED (METERS/SEC) \\
N & 1.610 & 2.028 & 3.272 & 3.268 & 1.677 & 1.176 & 1.194 \\
NNW & 1.590 & 1.833 & 2.938 & 2.700 & 1.362 & 1.146 & 1.107 \\
NW & 1.398 & 1.805 & 1.963 & 1.689 & 1.139 & 1.510 & 1.214 \\
WNW & 1.423 & 1.523 & 1.577 & 1.556 & 1.300 & 1.071 & 1.013 \\
W & 1.285 & 1.329 & 1.393 & 1.329 & 1.165 & 1.067 & 1.111 \\
WSW & 1.294 & 1.400 & 2.025 & 1.378 & 1.332 & 1.044 & 1.180 \\
SW & 1.426 & 1.775 & 2.676 & 3.221 & 1.443 & 1.273 & 1.081 \\
SSW & 1.561 & 1.957 & 3.395 & 3.460 & 1.573 & 1.231 & 1.268 \\
S & 1.510 & 1.608 & 2.160 & 2.013 & 1.619 & 1.633 & 1.305 \\
SSE & 1.810 & 2.310 & 3.495 & 2.358 & 1.491 & 1.261 & 1.197 \\
SE & 1.720 & 2.672 & 3.845 & 3.575 & 1.798 & 1.555 & 1.325 \\
ESE & 1.987 & 2.890 & 4.409 & 4.751 & 1.926 & 1.588 & 1.347 \\
E & 1.729 & 2.707 & 4.307 & 3.338 & 1.723 & 1.541 & 1.214 \\
ENE & 1.973 & 2.282 & 3.997 & 2.744 & 1.448 & 1.413 & 1.177 \\
NE & 1.982 & 2.140 & 3.007 & 2.128 & 1.389 & 1.333 & 1.162 \\
NNE & 1.731 & 2.211 & 2.680 & 1.968 & 1.611 & 1.115 & 1.092
\end{tabular}


FREQUENCX TABLES FOR 1989 ALL HOURS 8736 DATA POINTS KESSELRING SITE FREQUENCY OF EACH STABILITY CLASS FOR EACH WIND DIRECTION

\begin{tabular}{cccccccccc} 
WIND TOWARD & \multicolumn{7}{c}{ FRACTION OE TIME IN EACH STABILITY CLASS } \\
N & A & B & C & D & E & F & G & ALL \\
NNW & 0.2389 & 0.2340 & 0.1527 & 0.1897 & 0.0862 & 0.0690 & 0.0296 & 0.0465 \\
NW & 0.1625 & 0.2688 & 0.2021 & 0.2292 & 0.0583 & 0.0562 & 0.0229 & 0.0549 \\
WNW & 0.2818 & 0.2887 & 0.1134 & 0.1031 & 0.1100 & 0.0756 & 0.0275 & 0.0333 \\
W & 0.3120 & 0.2120 & 0.1040 & 0.1600 & 0.1080 & 0.0600 & 0.0440 & 0.0286 \\
WSW & 0.1574 & 0.1636 & 0.1543 & 0.2130 & 0.1821 & 0.0679 & 0.0617 & 0.0371 \\
SW & 0.1056 & 0.1767 & 0.1487 & 0.2522 & 0.1703 & 0.0948 & 0.0517 & 0.0531 \\
SSW & 0.0207 & 0.1271 & 0.2000 & 0.3519 & 0.1690 & 0.0791 & 0.0527 & 0.0738 \\
S & 0.0464 & 0.1000 & 0.0393 & 0.3250 & 0.2679 & 0.1357 & 0.0857 & 0.0321 \\
SSE & 0.0508 & 0.0621 & 0.0056 & 0.1356 & 0.3051 & 0.2316 & 0.2090 & 0.0203 \\
SE & 0.0688 & 0.0688 & 0.0212 & 0.1958 & 0.2328 & 0.1799 & 0.2328 & 0.0216 \\
ESE & 0.0345 & 0.0822 & 0.1008 & 0.3077 & 0.1645 & 0.1247 & 0.1857 & 0.0432 \\
E & 0.0165 & 0.0321 & 0.1251 & 0.5098 & 0.1551 & 0.0693 & 0.0920 & 0.1107 \\
ENE & 0.0183 & 0.0336 & 0.1103 & 0.6192 & 0.1281 & 0.0397 & 0.0508 & 0.2252 \\
NE & 0.0310 & 0.0877 & 0.2451 & 0.3981 & 0.1075 & 0.0696 & 0.0610 & 0.1331 \\
NNE & 0.1101 & 0.2026 & 0.1630 & 0.2159 & 0.1322 & 0.0903 & 0.0859 & 0.0520 \\
NNE & 0.1921 & 0.1623 & 0.1192 & 0.1987 & 0.1126 & 0.1424 & 0.0728 & 0.0346
\end{tabular}

\begin{tabular}{|c|c|c|c|c|c|c|c|}
\hline WIND & TOWARD & $\bullet$ & $\mathrm{RC}$ & $\begin{array}{l}\text { AVERAC } \\
\text { WIND }\end{array}$ & $\begin{array}{l}\text { WIND } \\
\text { SED (ME }\end{array}$ & $\begin{array}{l}\text { EEDS } \\
\text { SRS/SEC }\end{array}$ & \\
\hline & A & B & $C$ & $D$ & E & $\mathbf{F}$ & G \\
\hline$N$ & 1.52 .6 & 2.013 & 1.863 & 1.835 & 1.389 & 1.466 & 1.24 \\
\hline NNW & 1.391 & 2.152 & 2.669 & 2.667 & 1.410 & 1.400 & 1.22 \\
\hline NW & 1.436 & 1.663 & 1.762 & 1.622 & 1.400 & 1.179 & 1.172 \\
\hline WNW & 1.412 & 1.453 & 1.288 & 1.238 & 1.284 & 1.445 & 1.127 \\
\hline$W$ & 1.404 & 1.707 & 1.917 & 1.464 & 1.274 & 1.232 & 1.16 \\
\hline WSW & 1.444 & 1.852 & 2.102 & 1.974 & 1.551 & 1.353 & 1.18 \\
\hline SW & 1.573 & 2.103 & 2.707 & 2.545 & 1.456 & 1. .367 & 1.155 \\
\hline SSW & 1.211 & 1.763 & 1.858 & 1.462 & 1.233 & 1.389 & 1.140 \\
\hline$S$ & 1.136 & 1.499 & 1.000 & 1.389 & 1.082 & 1.049 & 2.17 \\
\hline SSE & 1.350 & 2.436 & 1.548 & 1.136 & 1.096 & 1.118 & 1.179 \\
\hline SE & 1.740 & 2.492 & 3.252 & 2.089 & 1.208 & 1.187 & 1.129 \\
\hline ESE & 1.568 & 2.191 & 3.798 & 2.036 & 1.275 & 1.210 & 1.161 \\
\hline $\mathbf{E}$ & 1.534 & 2.293 & 3.906 & 2.968 & 1.421 & 1.261 & 1.21 \\
\hline ENE & 1.741 & 2.357 & 3.495 & 2.669 & 1.585 & 1.385 & 1.260 \\
\hline $\mathrm{NE}$ & 1.710 & 2.187 & 2.533 & 2.218 & 1.402 & 1.268 & 1.286 \\
\hline NNE & 1.447 & 1.739 & 1.921 & 1.754 & 1.508 & 1.182 & 1.23 \\
\hline & & & & AGE WII & SPEEDS & & \\
\hline WIND & TOWARD & & & WIND & 2ED $\quad(M E$ & SEC & \\
\hline & A & B & C & D & $E$ & $E$ & $\mathbf{3}$ \\
\hline $\mathbf{N}$ & 1.703 & 2.322 & 2.461 & 2.764 & 1.531 & 1.621 & 1.33 \\
\hline NNW & 1.569 & 2.544 & 3.424 & 3.829 & 1.657 & 1.519 & 1.345 \\
\hline NW & 1.595 & 1.946 & 2.376 & 2.683 & 1.603 & 1.264 & 1.275 \\
\hline WNW & 1.553 & 1.700 & 1.596 & 1.538 & 1.433 & 1.580 & 1.218 \\
\hline $\boldsymbol{W}$ & 1.537 & 2.011 & 2.306 & 2.196 & 1.397 & 1.368 & 1.240 \\
\hline WSW & 1.614 & 2.259 & 2.712 & 2.531 & 1.768 & 1.502 & 1.25 \\
\hline SW & 1.738 & 2.491 & 3.593 & 3.204 & 1.681 & 1.520 & 1.25 \\
\hline SSW & 1.385 & 2.054 & 2.545 & 2.011 & 1.423 & 1.568 & 1.19 \\
\hline 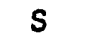 & 1.211 & 1.936 & 1.000 & 2.312 & 1.167 & 1.085 & 1.25 \\
\hline SSE & 1.623 & 2.877 & 2.300 & 1.373 & 1.184 & 1.229 & 1.26 \\
\hline $\mathrm{SE}$ & 1.985 & 2.981 & 4.253 & 3.645 & 1.448 & 1.379 & 1.18 \\
\hline ESE & 1.813 & 2.832 & 4.510 & 3.754 & 1.497 & 1.390 & 1.24 \\
\hline $\mathbf{E}$ & 1.769 & 2.818 & 4.540 & 4.557 & 1.648 & 1.421 & 1.30 \\
\hline ENE & 1.997 & 2.803 & 4.061 & 3.695 & 1.885 & 1.609 & 1.36 \\
\hline NE & $1 . \bar{y} \bar{z} \bar{Z}$ & 2.500 & 3.170 & 3.278 & 1.618 & $1: 398$ & 1.428 \\
\hline NNE & 1.603 & 2.086 & 2.517 & 2.258 & 1.682 & 1.274 & 1.39 \\
\hline
\end{tabular}


FREQUENCY TABLES FOR 1983 ALL HOURS 8759 DATA POINTS WINDSOR SITE FRENUENCY OF EACH STABILITY CLASS FOR EACH WIND DIRECTION WIND TOWARD FRACTION OF TIME IN EACH STABILITY CLASS

$\begin{array}{lccccccccc} & \text { A } & \text { B } & C & \text { D } & \mathbf{E} & \mathbf{F} & \mathbf{G} & \text { ALL } \\ \text { N } & 0.1654 & 0.0888 & 0.1021 & 0.1964 & 0.3019 & 0.0522 & 0.0932 & 0.1029 \\ \text { NNW } & 0.2742 & 0.1018 & 0.0705 & 0.1253 & 0.11984 & 0.0757 & 0.1540 & 0.0437 \\ \text { NW } & 0.2930 & 0.0806 & 0.0879 & 0.1429 & 0.1062 & 0.0806 & 0.2088 & 0.0312 \\ \text { WNW } & 0.3516 & 0.0989 & 0.0714 & 0.0495 & 0.1429 & 0.0879 & 0.1978 & 0.0208 \\ \text { W } & 0.3077 & 0.1231 & 0.0615 & 0.0821 & 0.1436 & 0.0564 & 0.2256 & 0.0223 \\ \text { WSW } & 0.2941 & 0.1629 & 0.1357 & 0.0905 & 0.0950 & 0.0860 & 0.1357 & 0.0252 \\ \text { SW } & 0.2140 & 0.0996 & 0.1845 & 0.1181 & 0.1882 & 0.0664 & 0.1292 & 0.0309 \\ \text { SSW } & 0.1250 & 0.1039 & 0.2306 & 0.1849 & 0.1180 & 0.0616 & 0.1761 & 0.0648 \\ \text { S } & 0.0985 & 0.0913 & 0.1876 & 0.2344 & 0.1733 & 0.0546 & 0.1603 & 0.1588 \\ \text { SSE } & 0.1346 & 0.0850 & 0.1442 & 0.1615 & 0.1927 & 0.0689 & 0.2131 & 0.1061 \\ \text { SE } & 0.0901 & 0.0732 & 0.1625 & 0.3047 & 0.1843 & 0.0556 & 0.1296 & 0.1356 \\ \text { ESE } & 0.119 & 0.0867 & 0.1300 & 0.2915 & 0.1599 & 0.0762 & 0.1360 & 0.0764 \\ \text { E } & 0.1699 & 0.0919 & 0.0891 & 0.2006 & 0.1894 & 0.0585 & 0.2006 & 0.0410 \\ \text { ENE } & 0.1623 & 0.0830 & 0.1283 & 0.2226 & 0.0943 & 0.0755 & 0.2340 & 0.0303 \\ \text { NE } & 0.1635 & 0.1099 & 0.1475 & 0.1796 & 0.1501 & 0.0509 & 0.1984 & 0.0426 \\ \text { NNE } & 0.1574 & 0.1303 & 0.1201 & 0.2352 & 0.1861 & 0.0355 & 0.1354 & 0.0675\end{array}$

NNE $\quad 0.1574 \quad 0.1303 \quad 0.1201 \quad 0.2352 \quad 0.1861 \quad 0.03550 .1354$

\begin{tabular}{|c|c|c|c|c|c|c|c|}
\hline \multirow{3}{*}{ WIND } & \multirow{2}{*}{\multicolumn{2}{|c|}{ TOWARD }} & \multicolumn{5}{|c|}{ RECIPROCAL-AVERAGED WIND SPEEDS } \\
\hline & & & & WIN & SPEED & (METERS) & EC! \\
\hline & A & B & c & $D$ & E & $\boldsymbol{E}$ & G \\
\hline $\mathrm{N}$ & 1.603 & .263 & .427 & 2.329 & 1.404 & 1.548 & 1.381 \\
\hline $\mathrm{N} N \mathrm{WW}$ & 1.669 & 74 & 444 & 861 & 1.437 & 1.712 & 1.382 \\
\hline NW & 1.412 & 14 & .406 & .059 & 1.402 & 1.695 & 1.271 \\
\hline WNW & 1.484 & 34 & 1.724 & .607 & 1.588 & 1.754 & 1.303 \\
\hline W & 1.496 & 82 & 1.870 & 562 & & & 1.267 \\
\hline WSW & 7 & 2 & .768 & .449 & .553 & 1.774 & 1.433 \\
\hline SW & 1.4 & 36 & 2.007 & .874 & $i$. & 1.989 & 1.286 \\
\hline SSW & 1.4 & 9 & 2.660 & 2.415 & $\therefore .542$ & 1.805 & 1.413 \\
\hline$s$ & 1 & .5 & 2.750 & 2.854 & $\ldots 755$ & 1. & 1.362 \\
\hline SSE & 8 & & & .114 & 1.454 & & \\
\hline$S E$ & 1 & & & 2 & & & 1.276 \\
\hline ESE &.$\epsilon$ & 7 & & .5610 & & & 1.288 \\
\hline $\mathbf{E}$ & 10 & 3 & & 1.455 & 91 & 1.535 & 1.224 \\
\hline ENE & 1. &.$?$ & 2. & 2.019 & & 1. & 1.235 \\
\hline $\mathrm{NE}$ & , & & 2.591 & 2.150 & 360 & 1.432 & 1.171 \\
\hline NNE & 6 & & & 1.838 & 1.341 & 1.313 & 1.276 \\
\hline & & & & 4 & 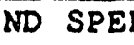 & & \\
\hline SNL & & & & & & (C) & \\
\hline & A & B & C & D & $E$ & $E$ & G \\
\hline $\mathbf{N}$ & 1.816 & 2.673 & 3.093 & 3.193 & 1.565 & 1.764 & 1.494 \\
\hline NNW & 1.885 & 2.874 & 3.030 & 2.900 & 1.642 & 2.021 & 1.515 \\
\hline$N W$ & 1. & & & 1.179 & & 2. & 1.407 \\
\hline WNW & 6 & 2. & 2.854 & 2.367 & 2. & 2.081 & 1.411 \\
\hline$w$ & 1 & 2. & 2. & 2.319 & 1.829 & 1.891 & 1.373 \\
\hline WSW & 0 & 2.7 & 2.103 & 2.955 & 1.895 & 2.021 & 1.590 \\
\hline SW & 1.614 & 2.2 & 2.562 & 2.719 & 1.969 & 2.228 & 1.377 \\
\hline SSW & 1.6 & & & 3.313 & & 2.114 & 1.55 \\
\hline$S$ & 18 & & & 3.468 & & 2. & 1.48 \\
\hline SSE & 1.7 & 2.5 & 3. & 2.8 & & 2.005 & 1.439 \\
\hline SE & 2 & 3.2 & 4.062 & 3.725 & 1.779 & 1.920 & 1.388 \\
\hline ESE & 1.850 & 3.126 & 4.100 & 3.898 & & 1.788 & 1.414 \\
\hline$E$ & 1.872 & 2.9 & & & 1.3 & 1.776 & 1.328 \\
\hline ENE & 1.8 & 3. & & & & 1.850 & 1.334 \\
\hline $\mathrm{NE}$ & 1.8 & 3.2 & & & & 1.616 & 1.258 \\
\hline NNE & 1.877 & 2.979 & 3.389 & 2.568 & 1.551 & 1.505 & 1.370 \\
\hline
\end{tabular}




\section{APPENDIX B \\ WIND ROSES}




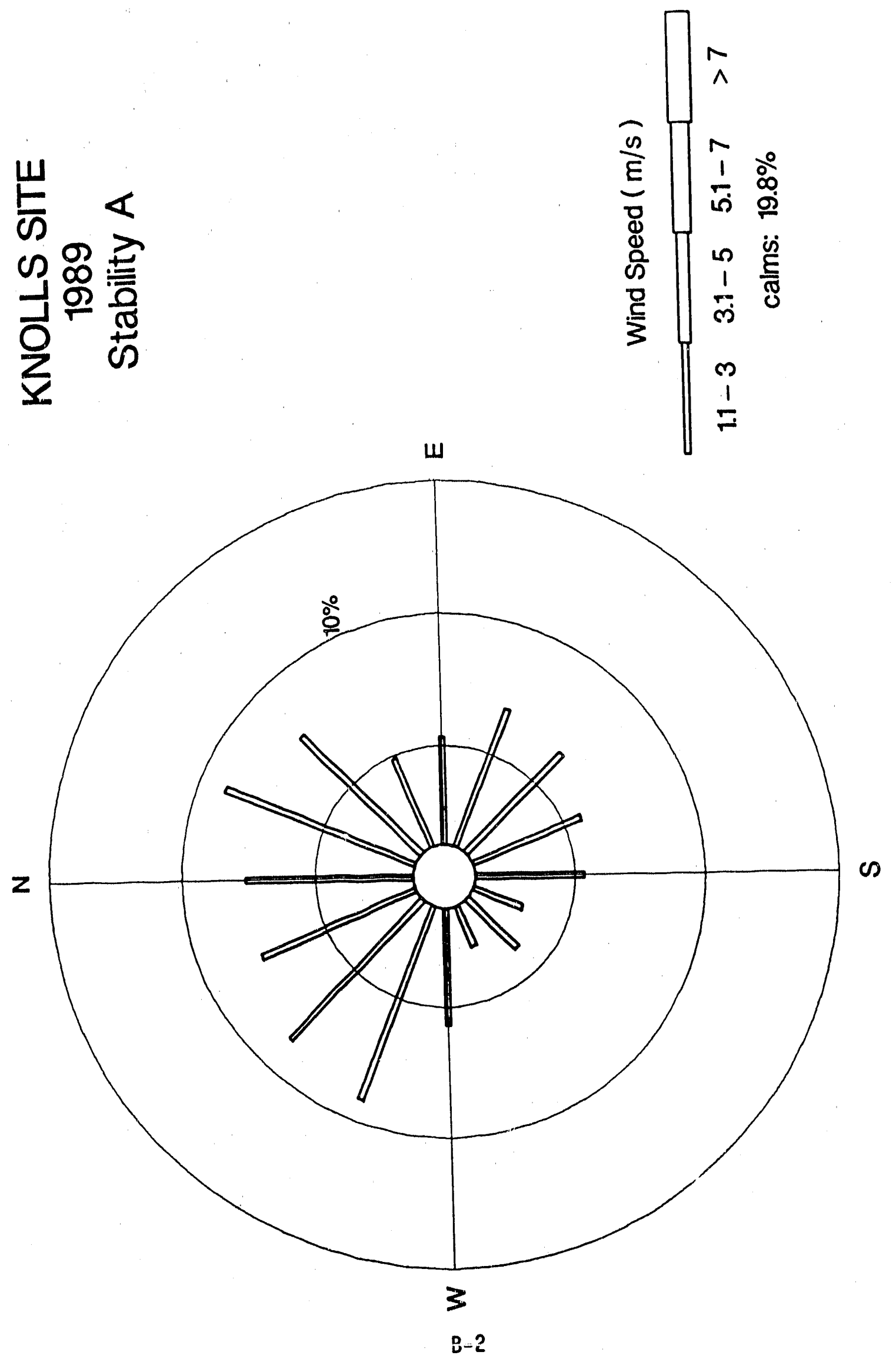




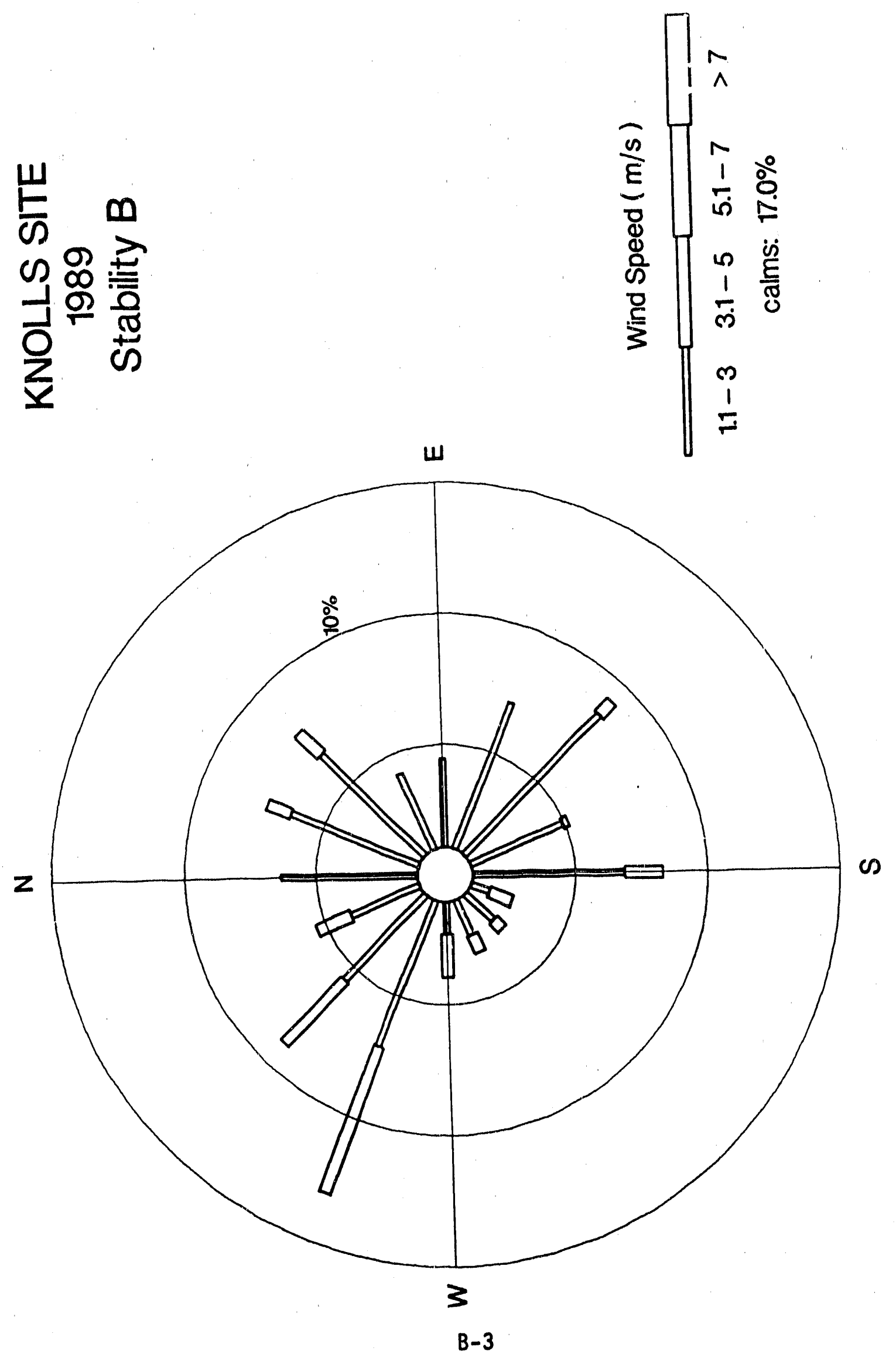




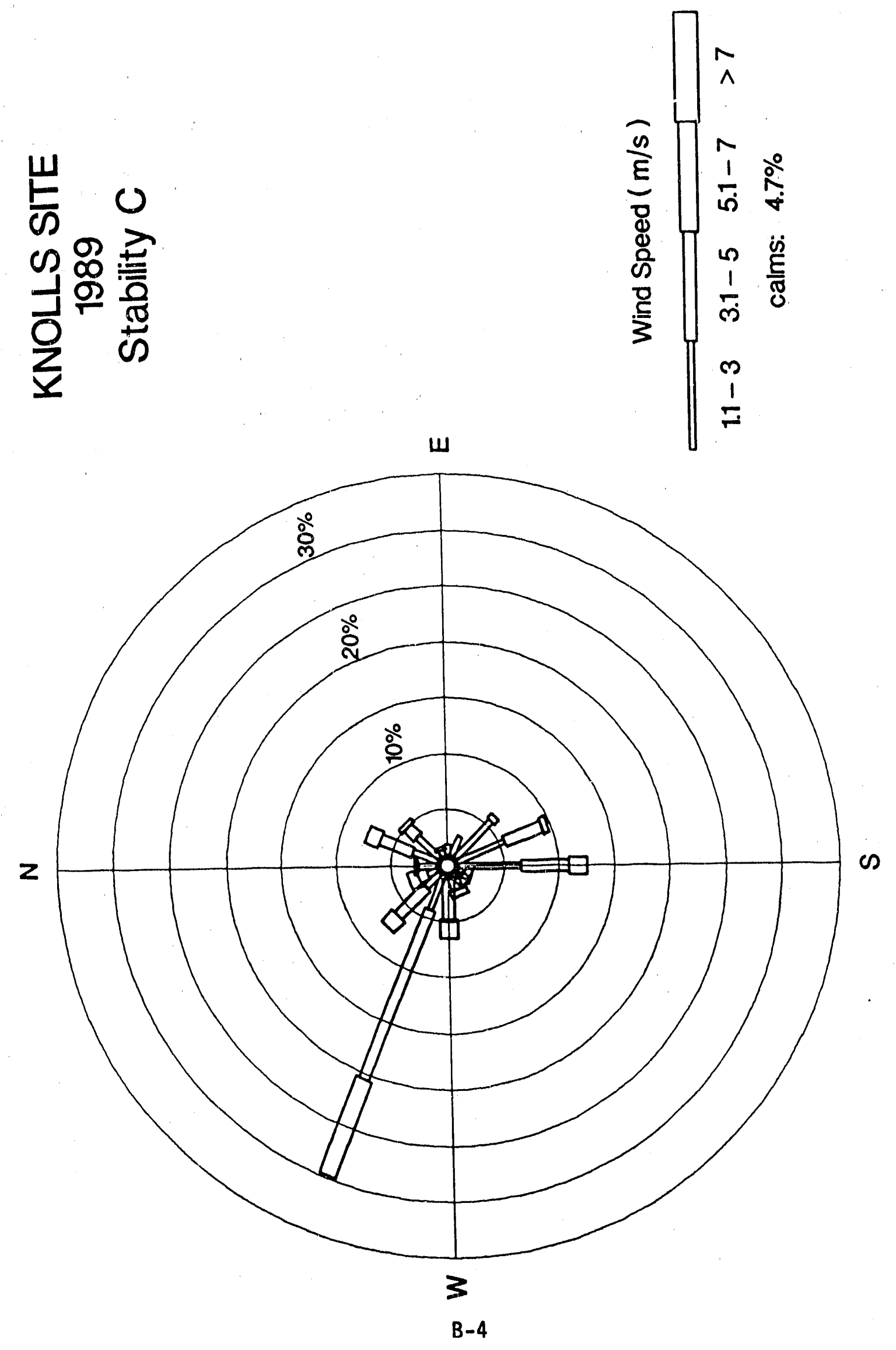




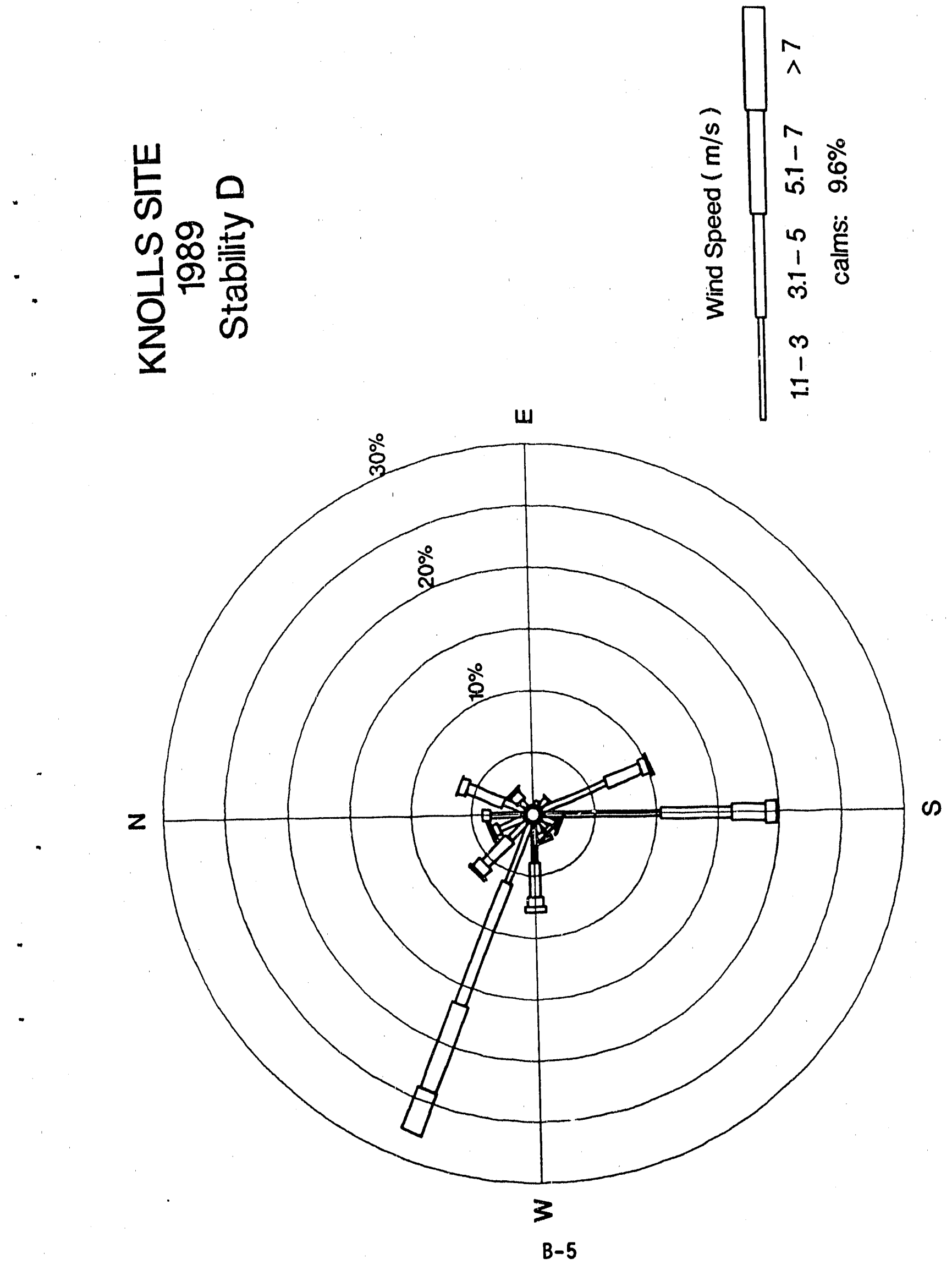




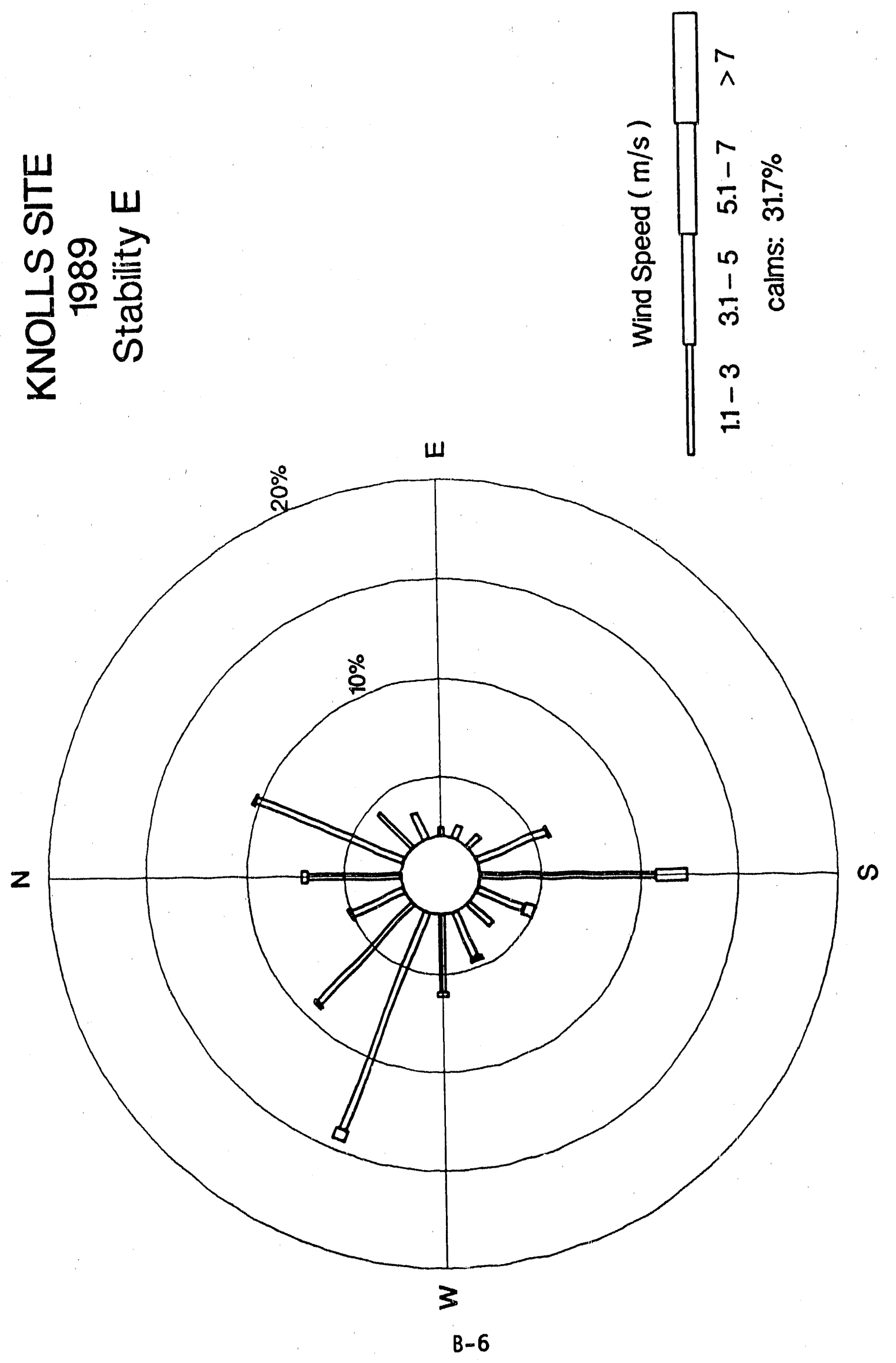




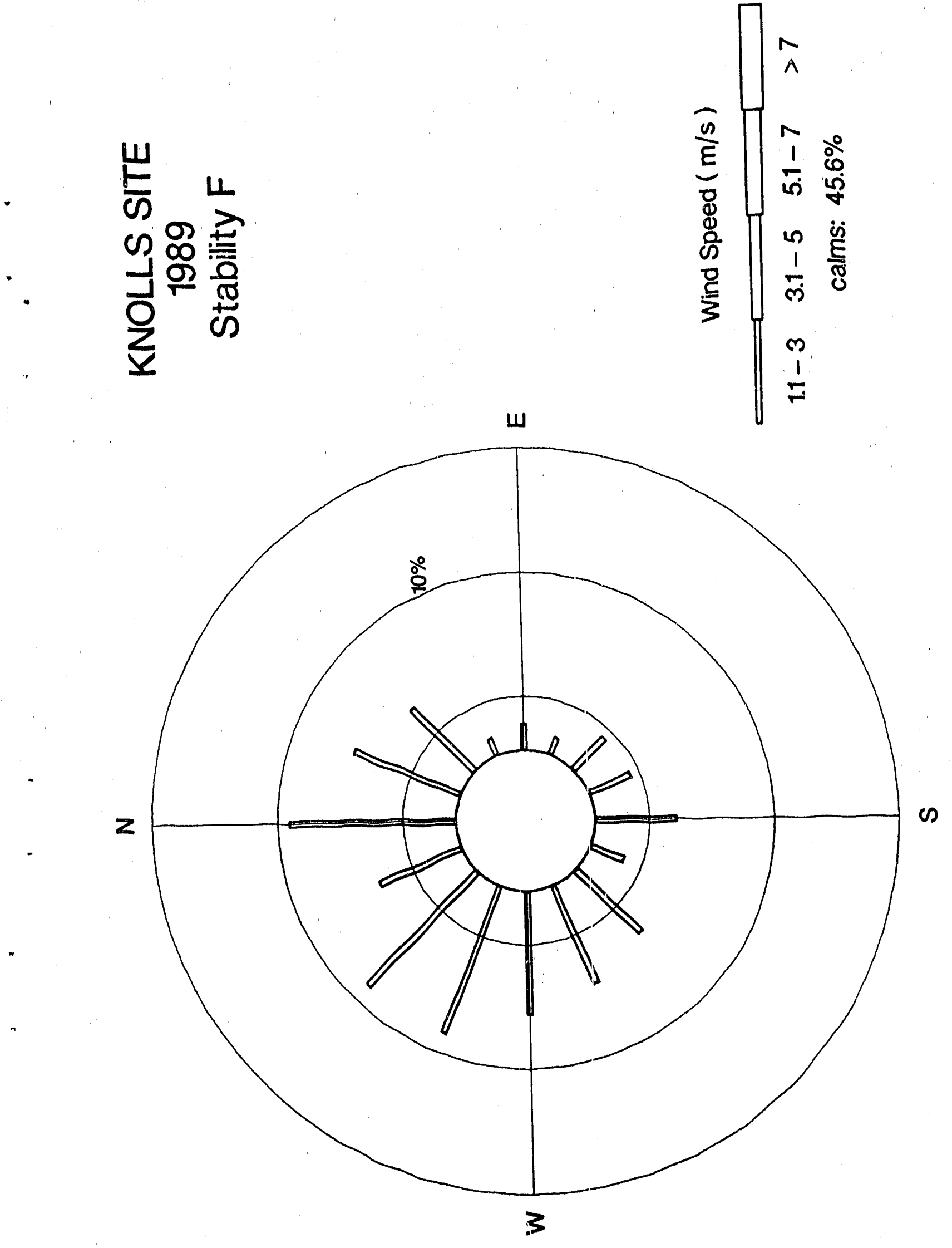




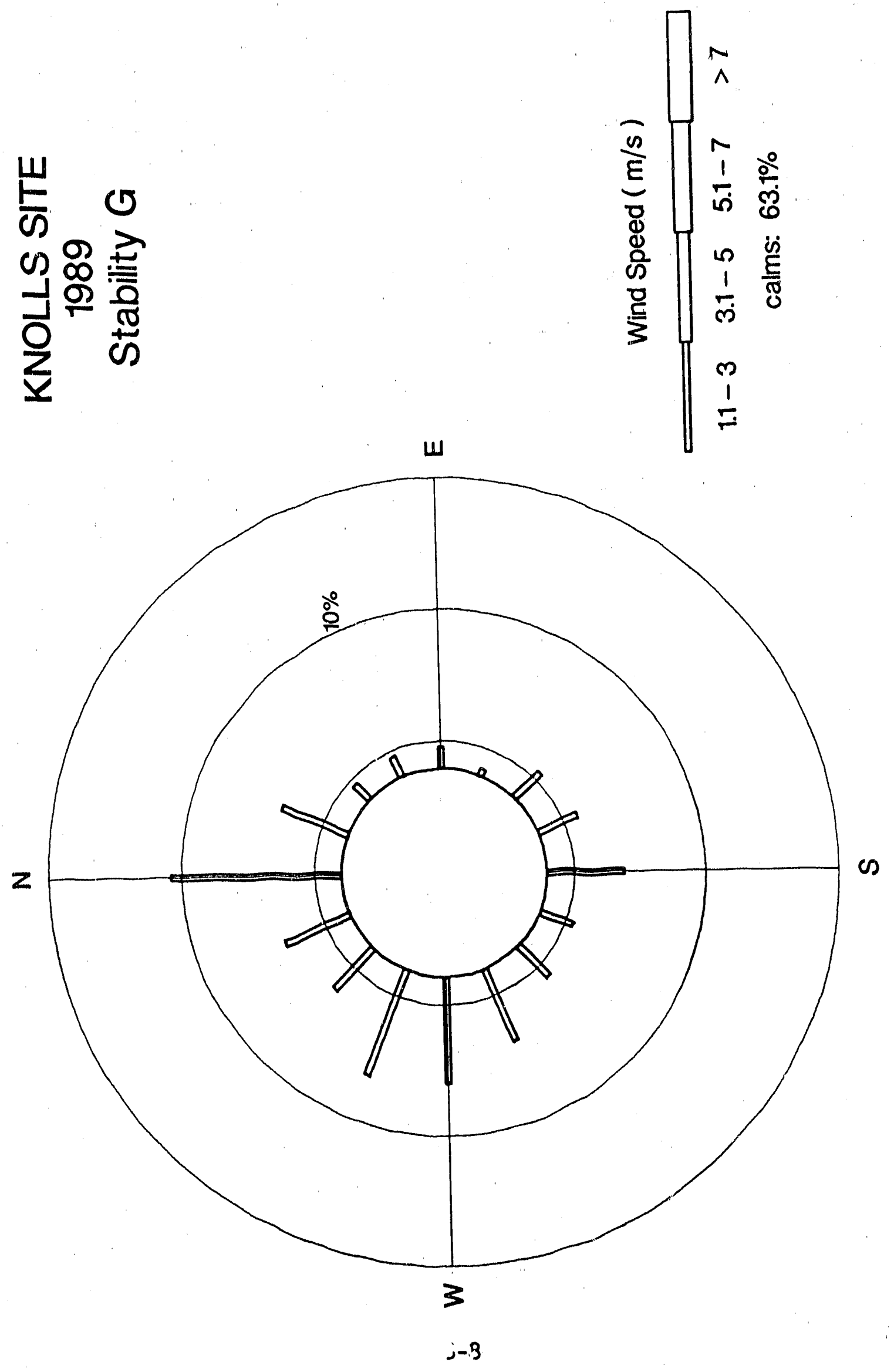




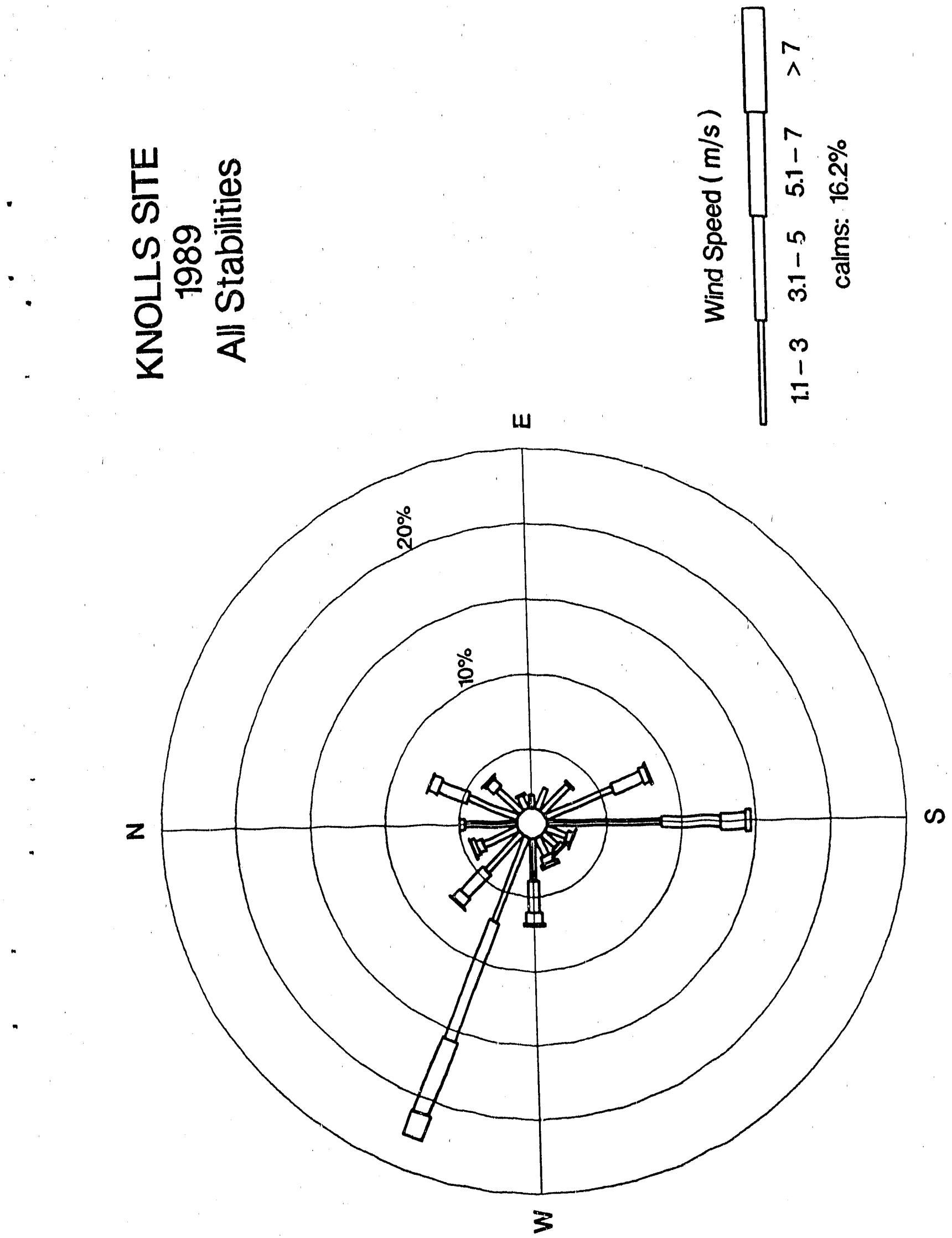



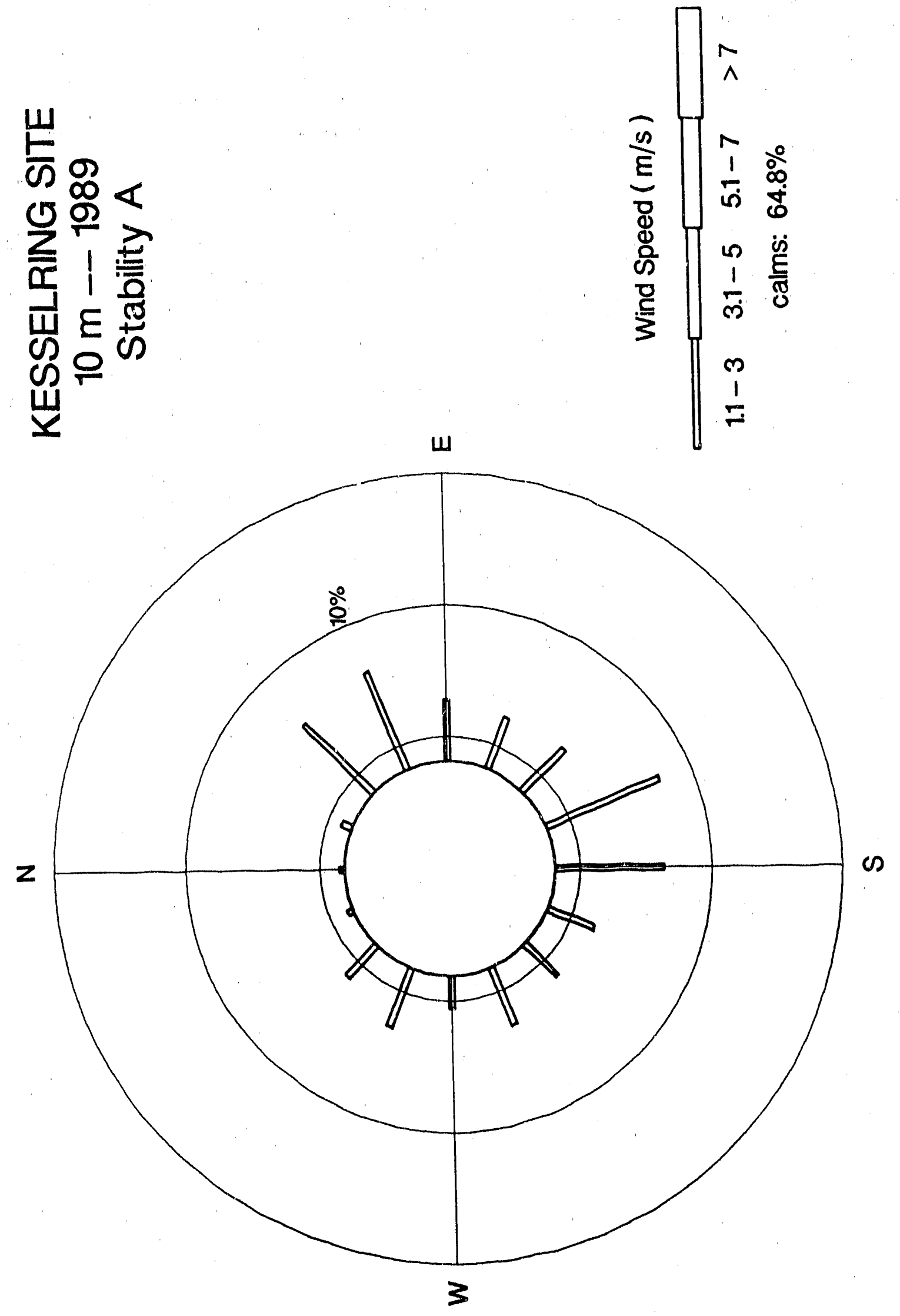

B-10 


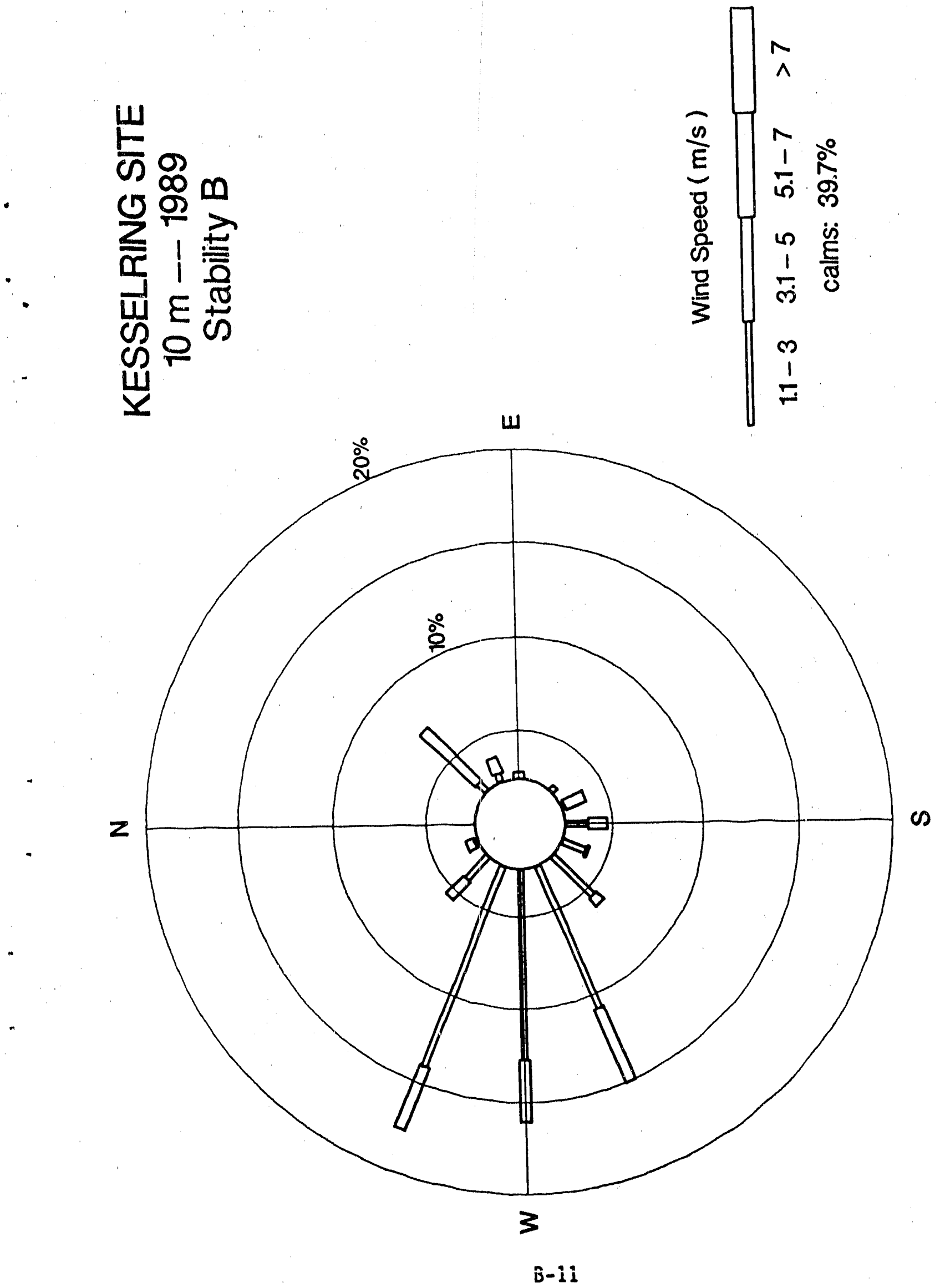



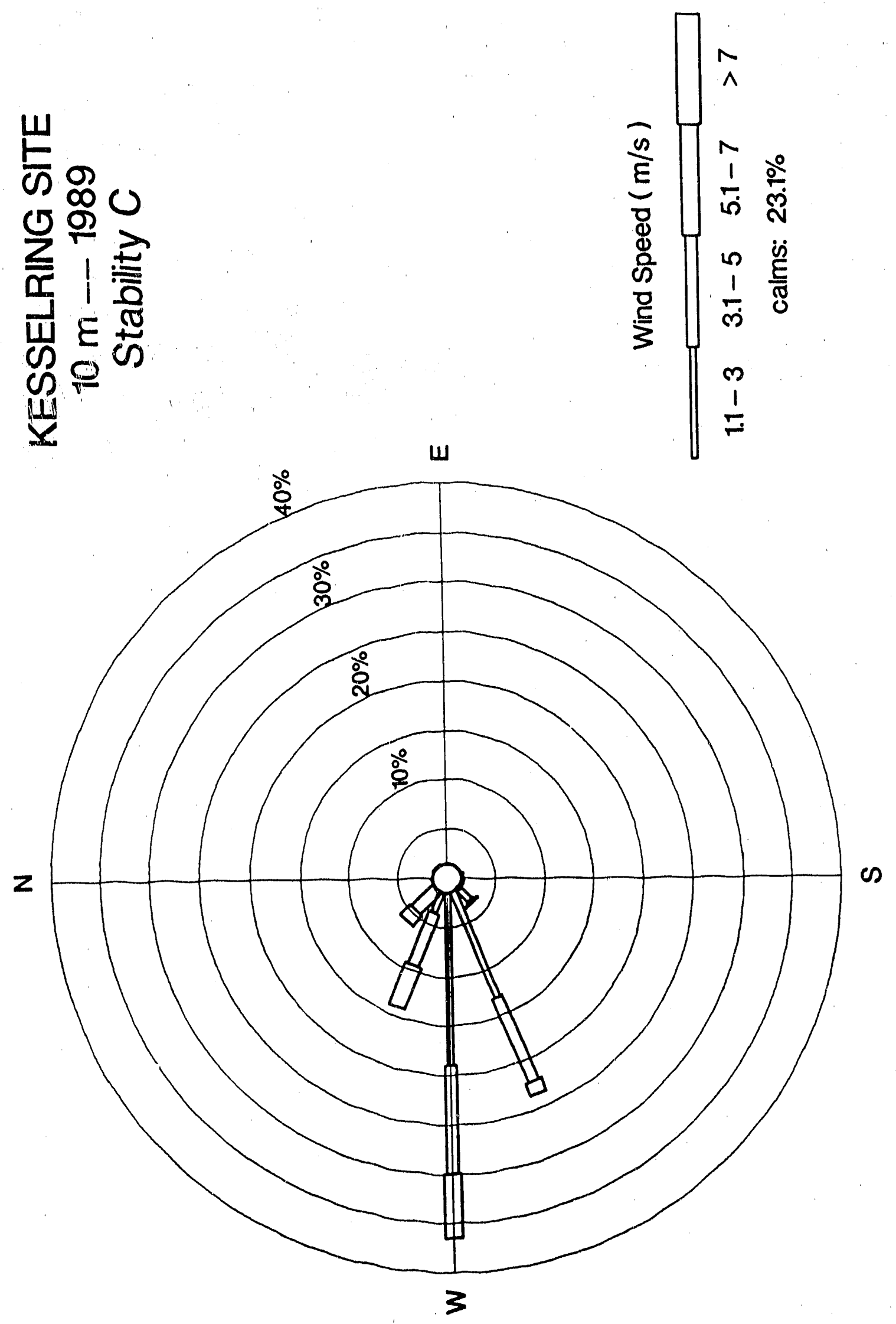


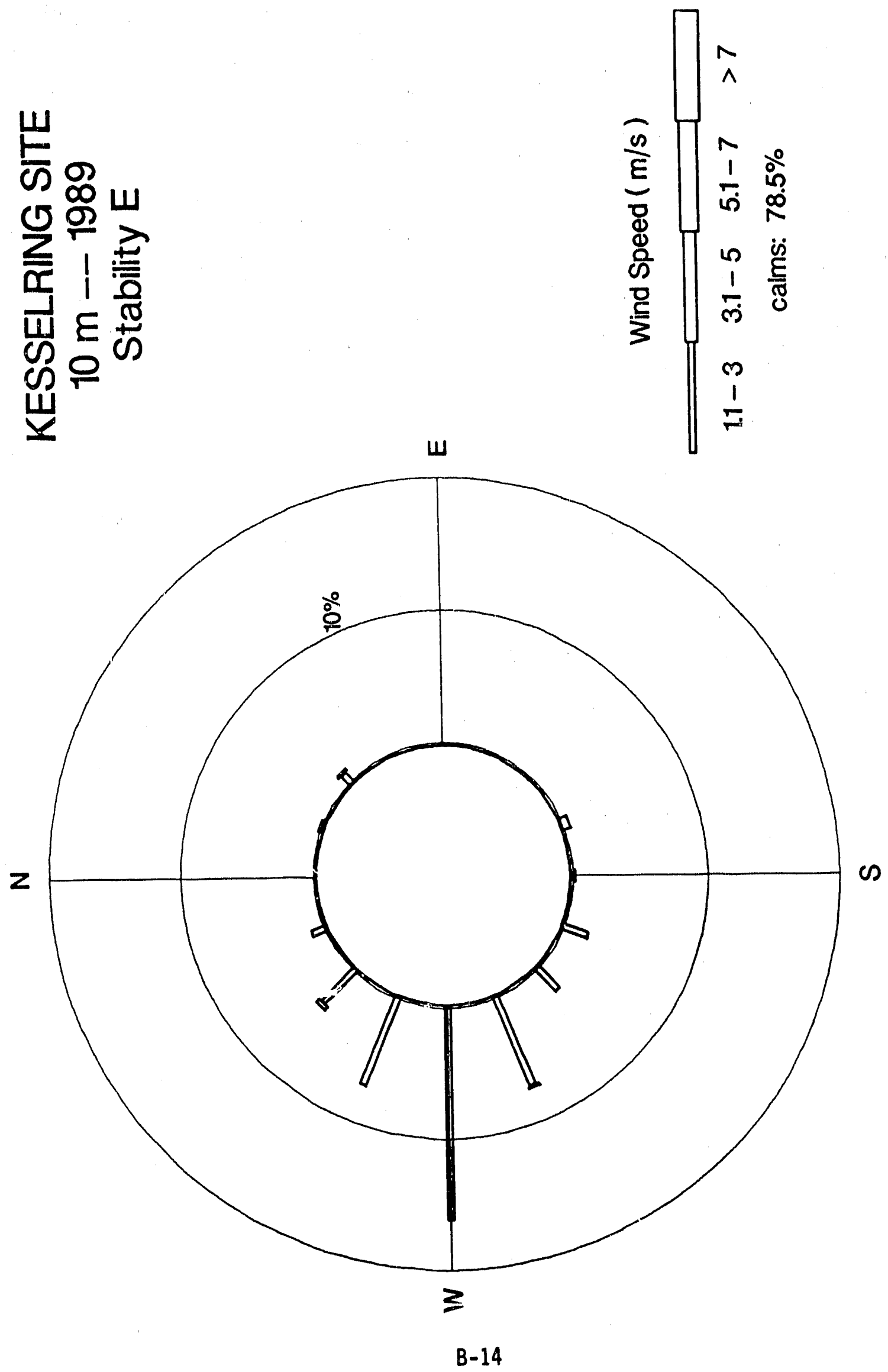




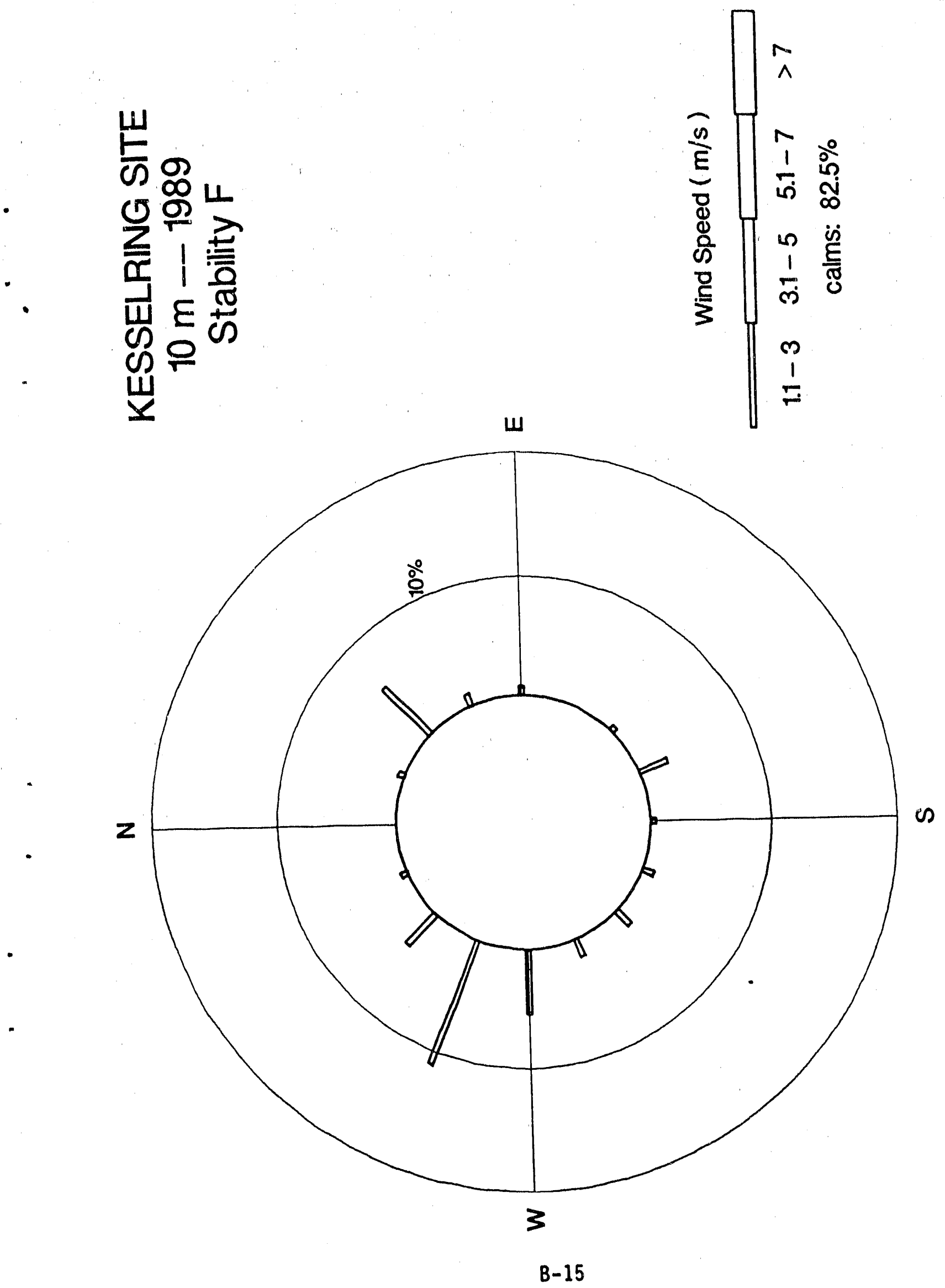




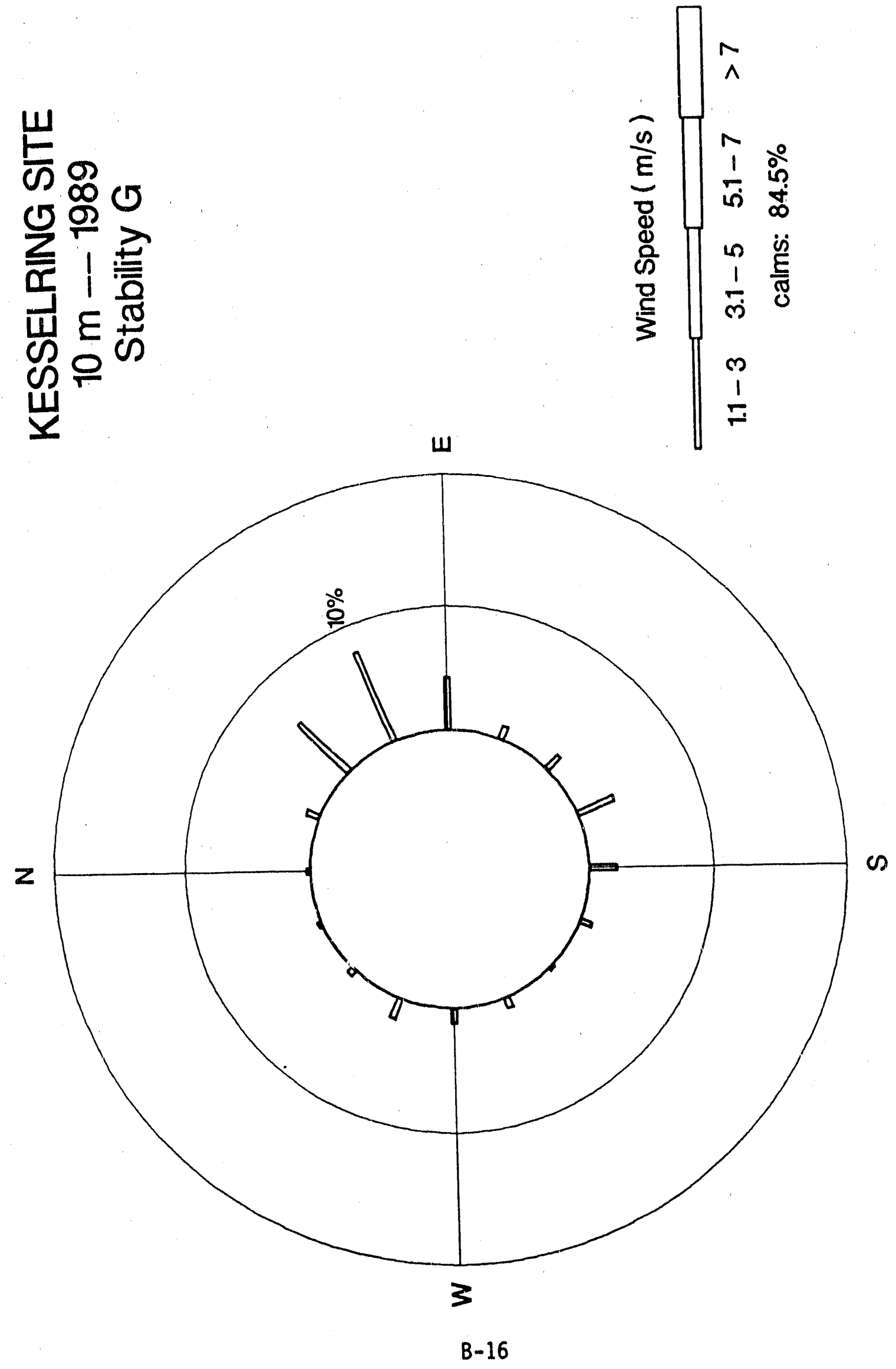




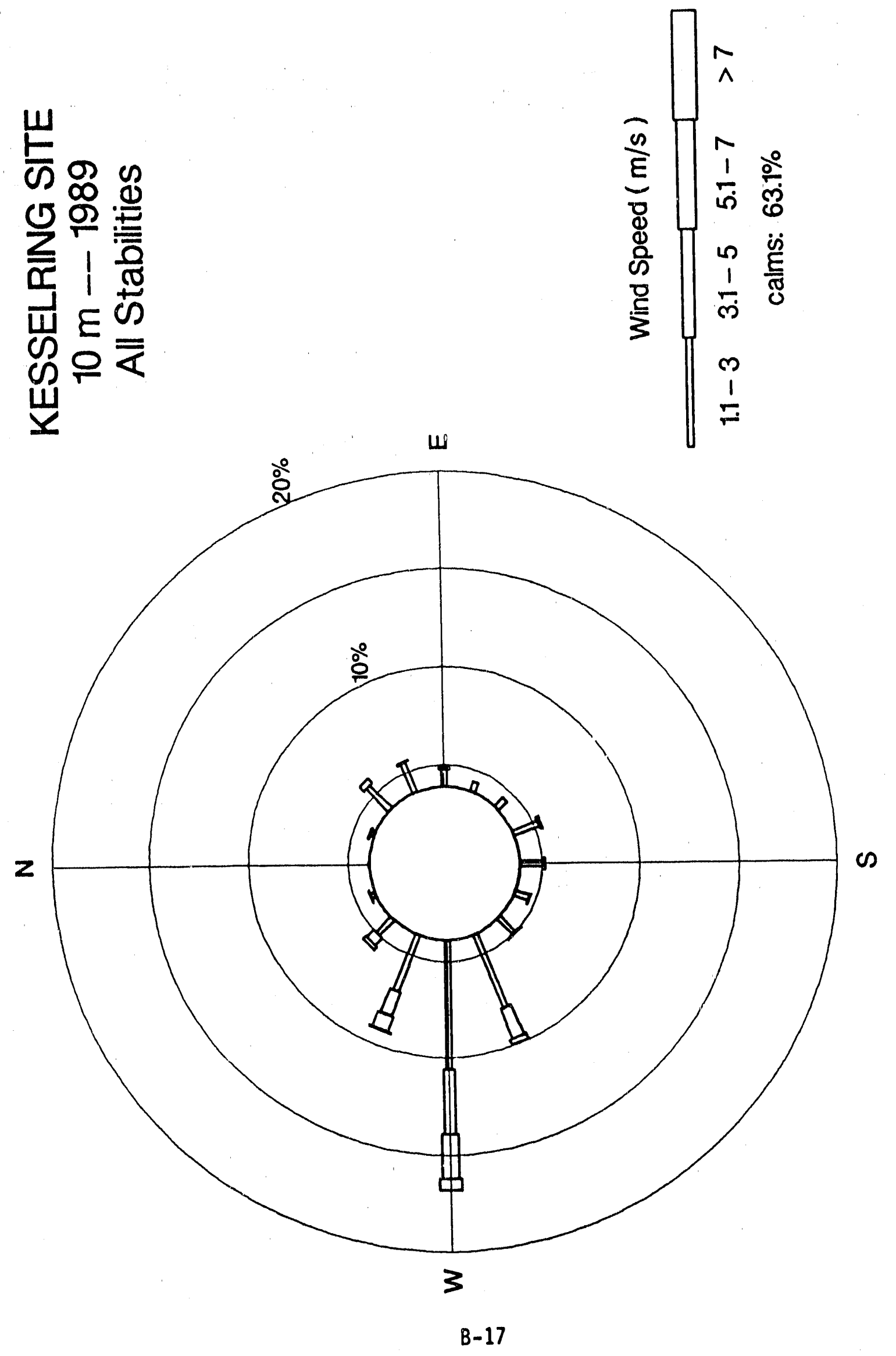




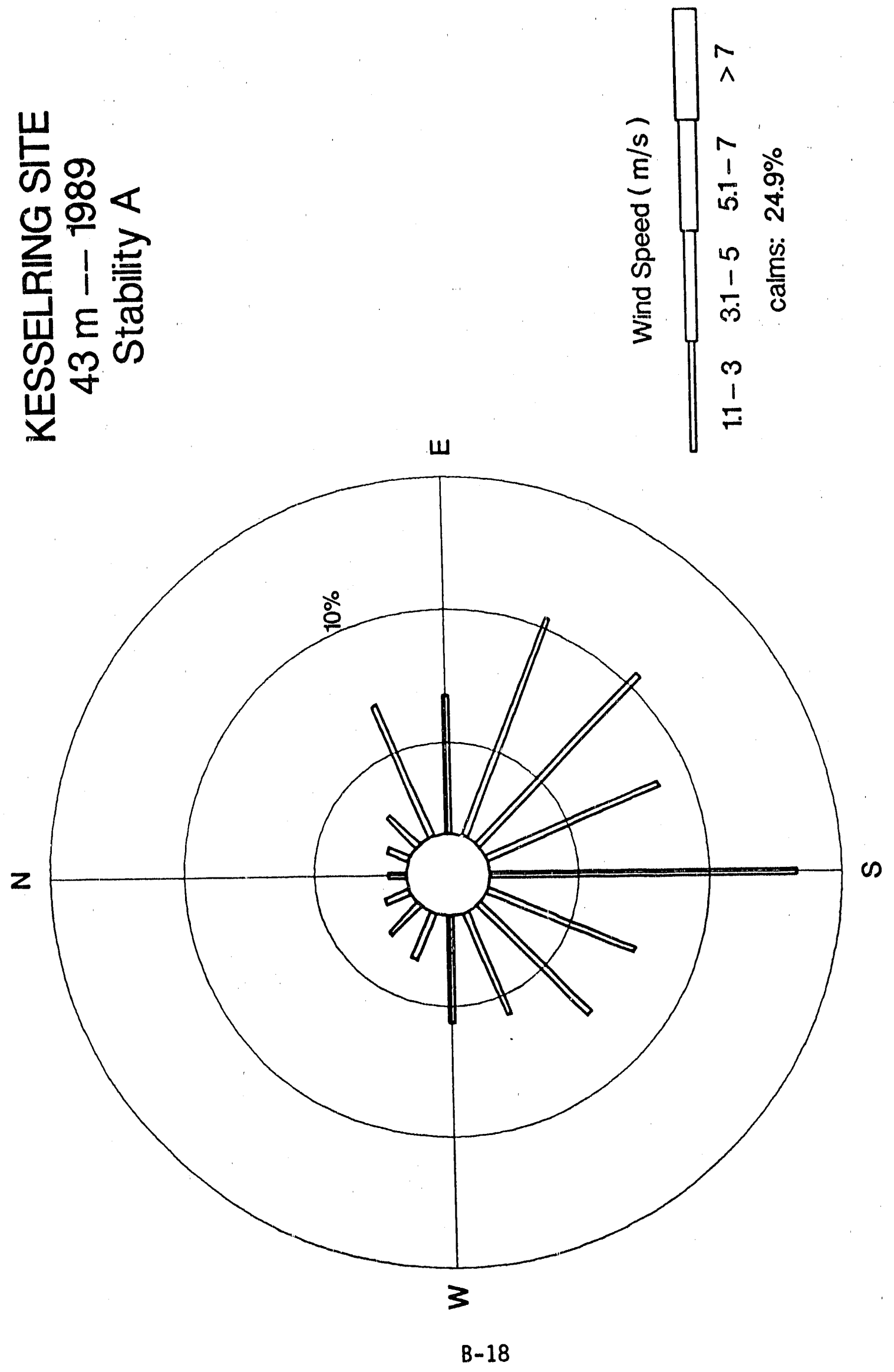




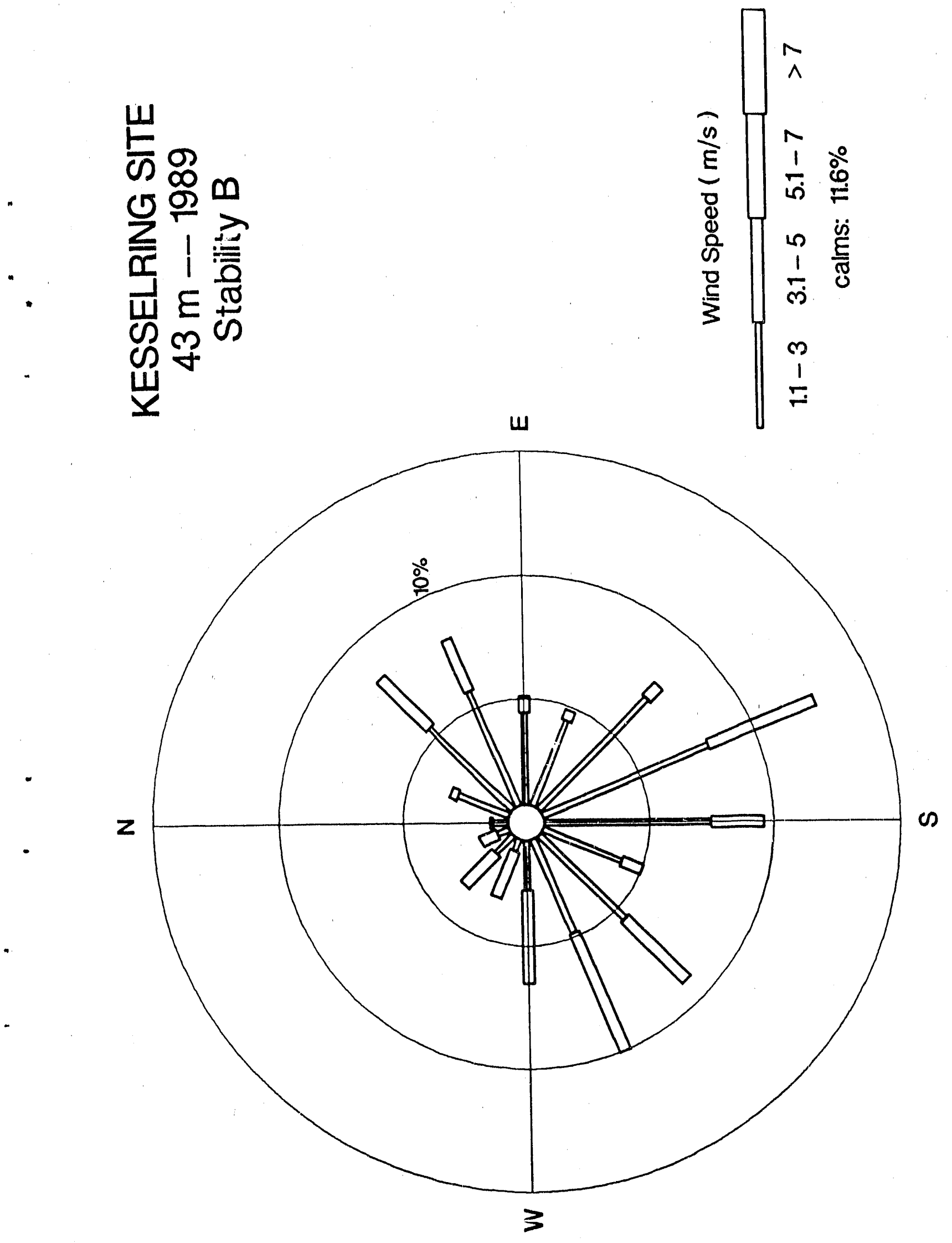

B-19 


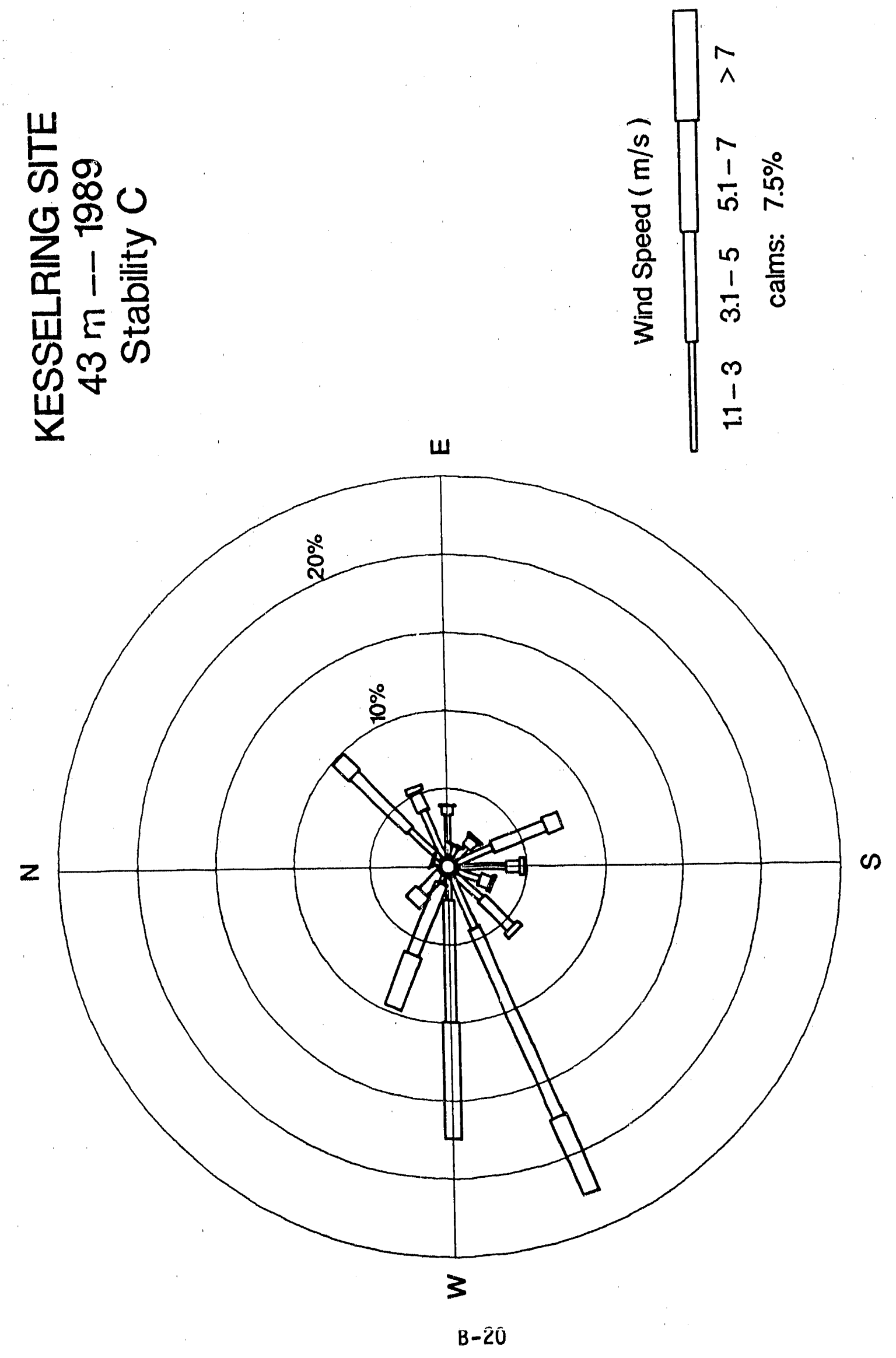




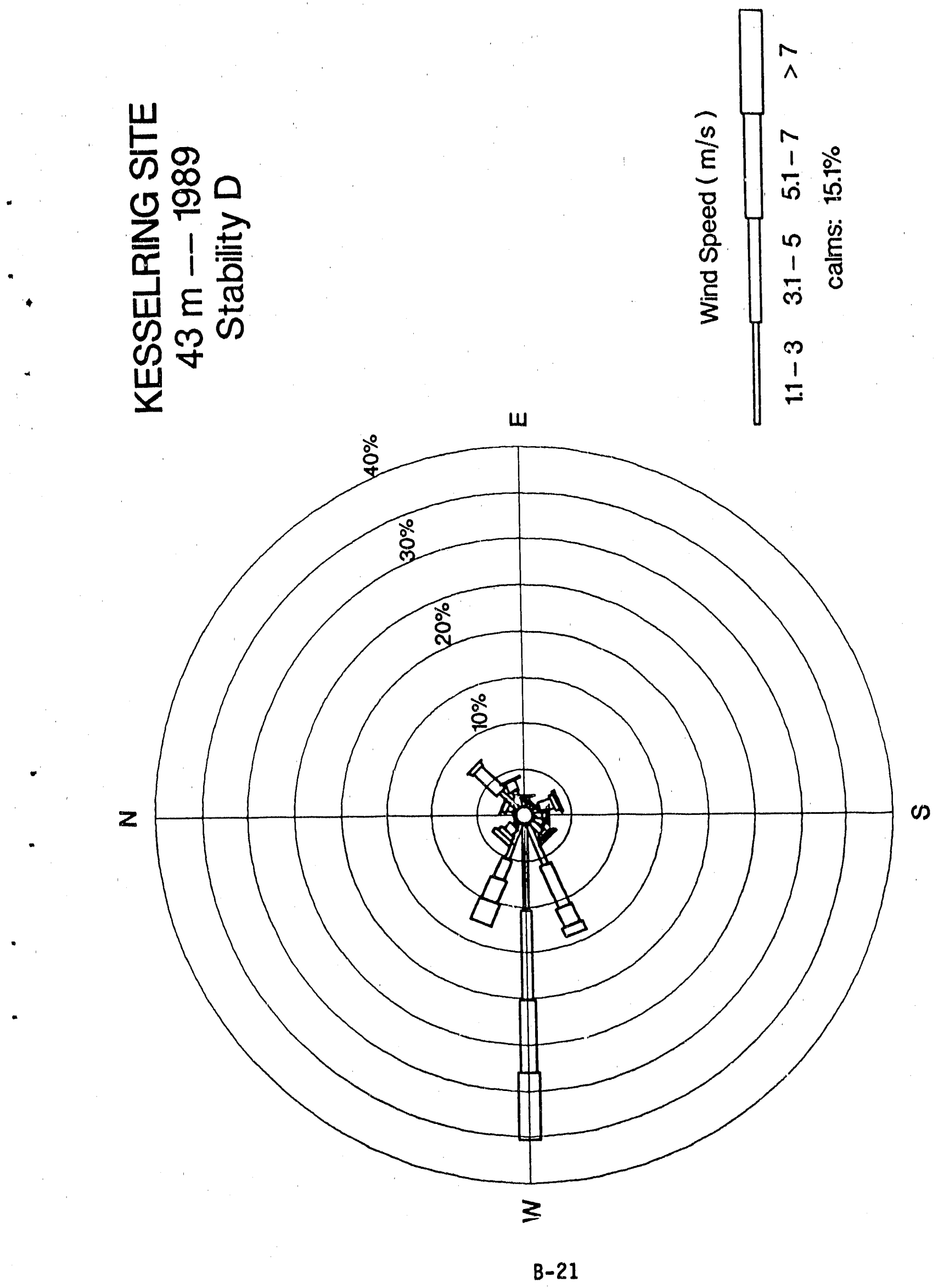




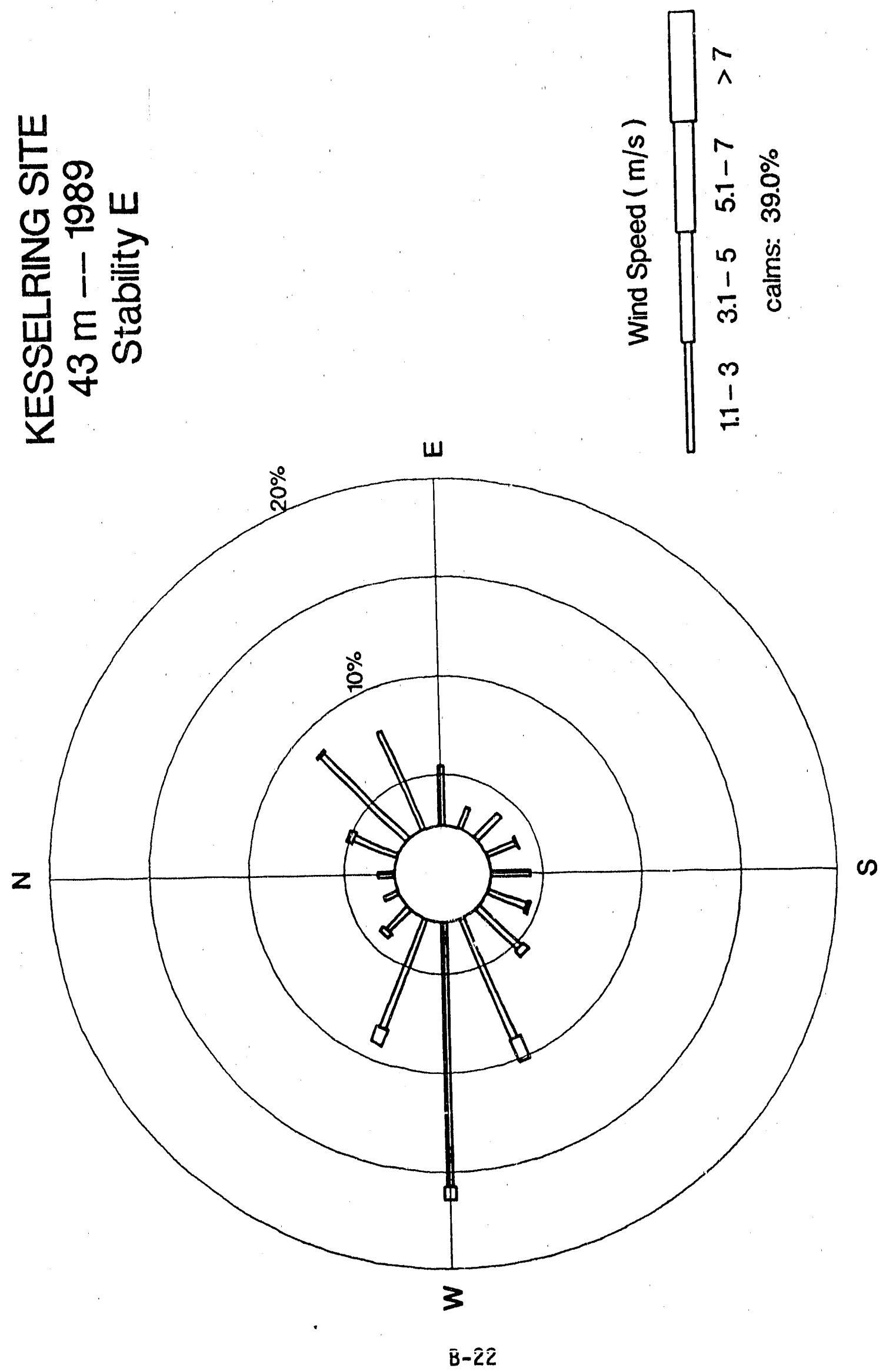




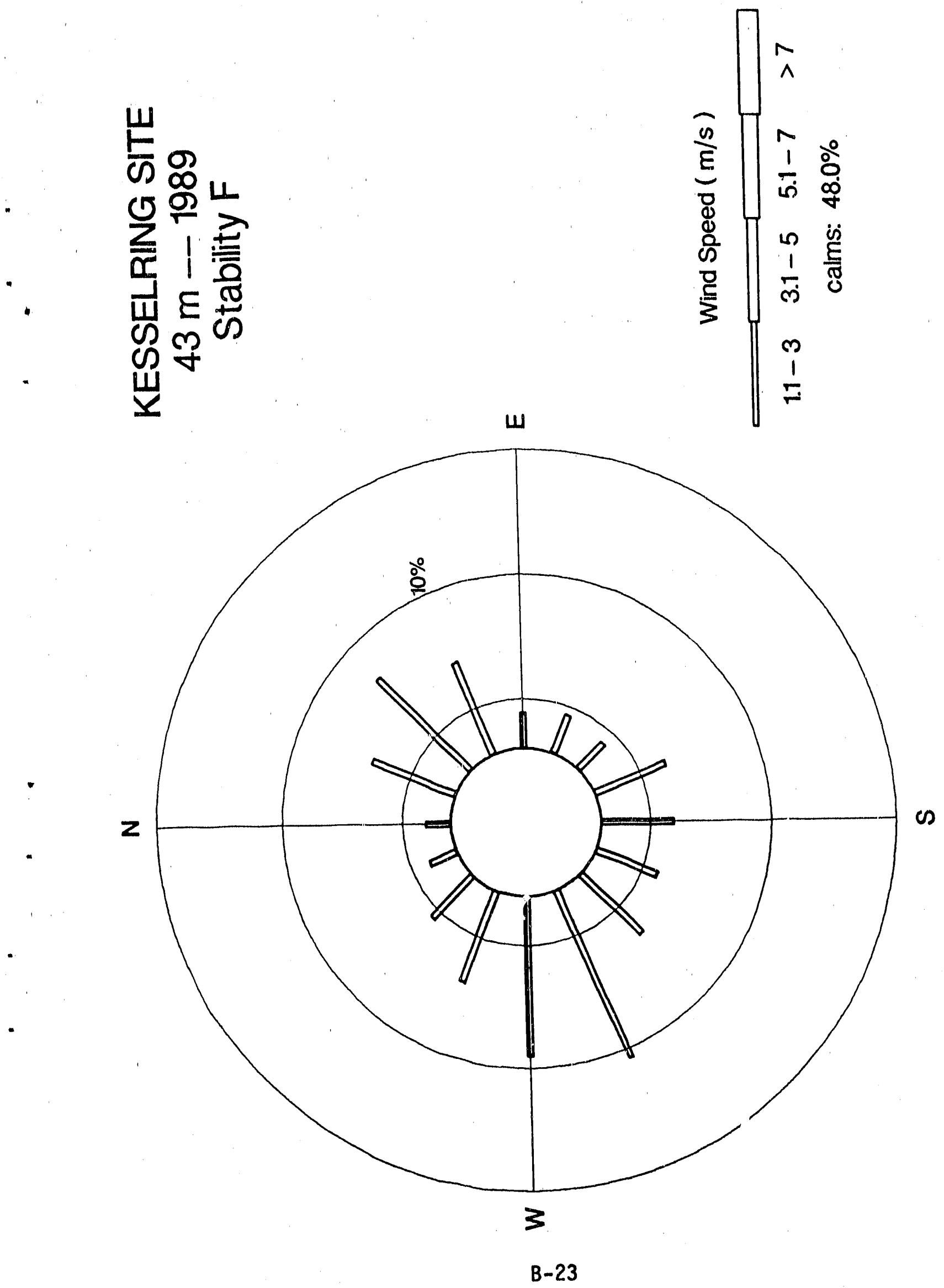




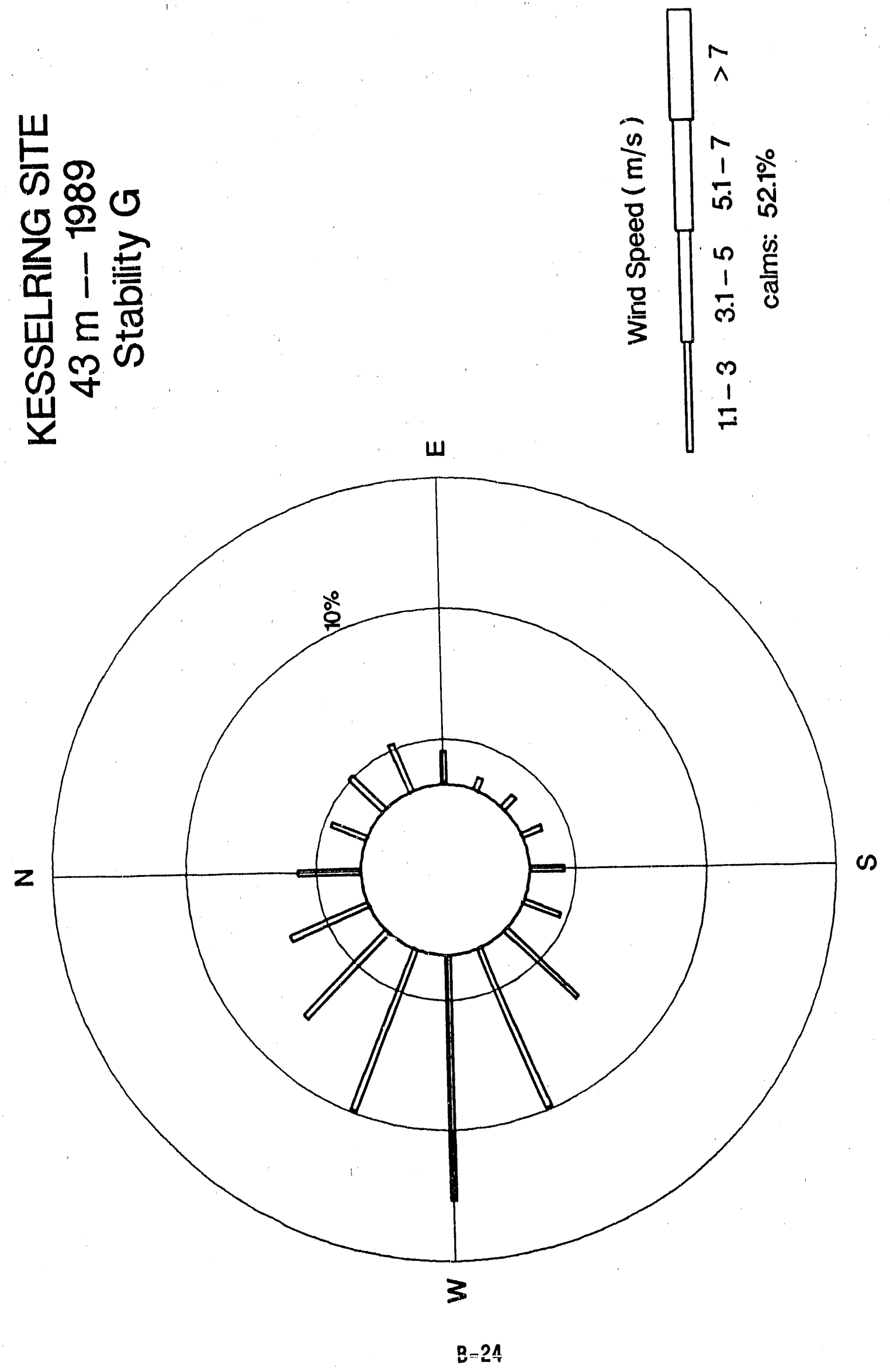




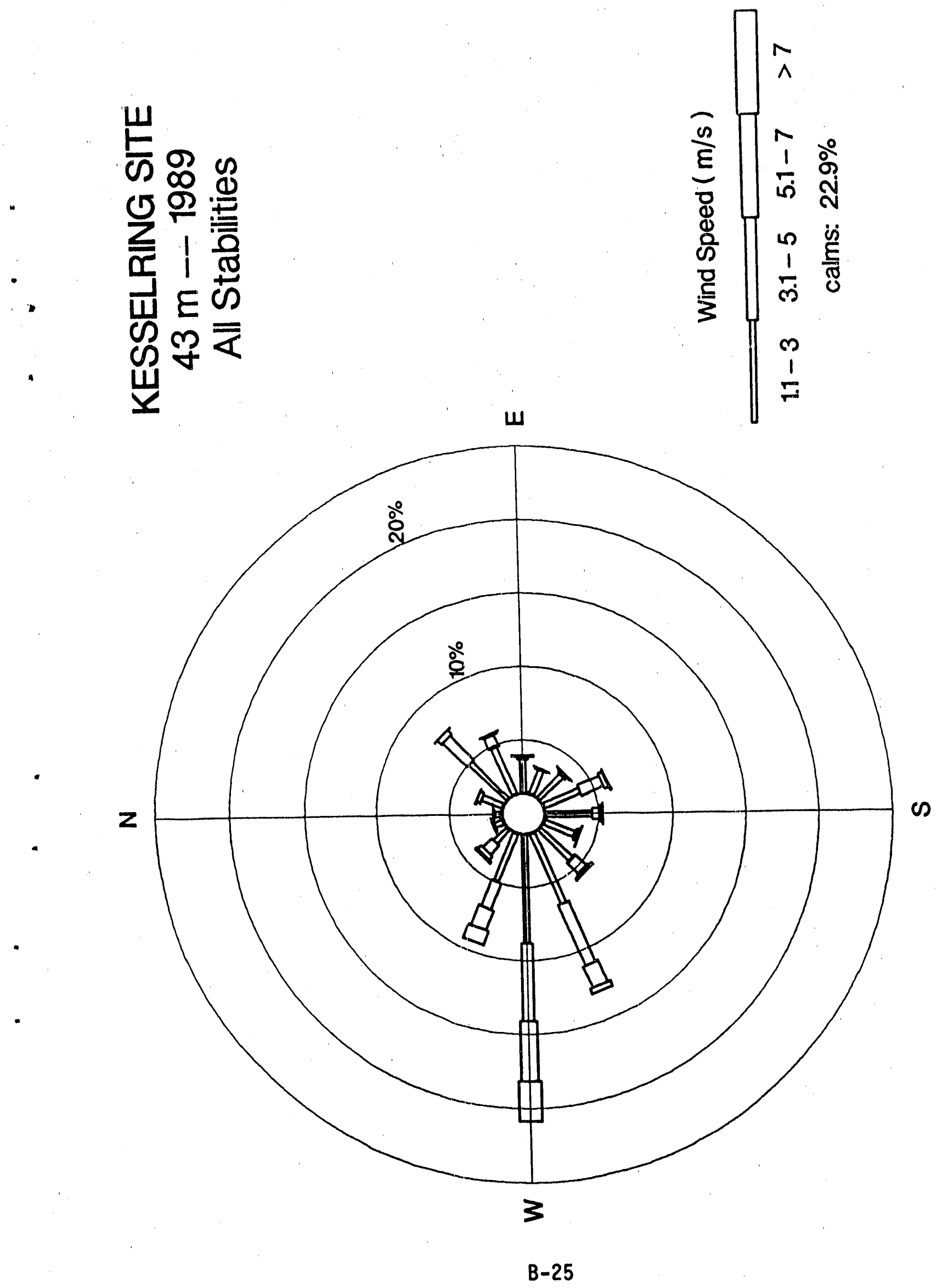




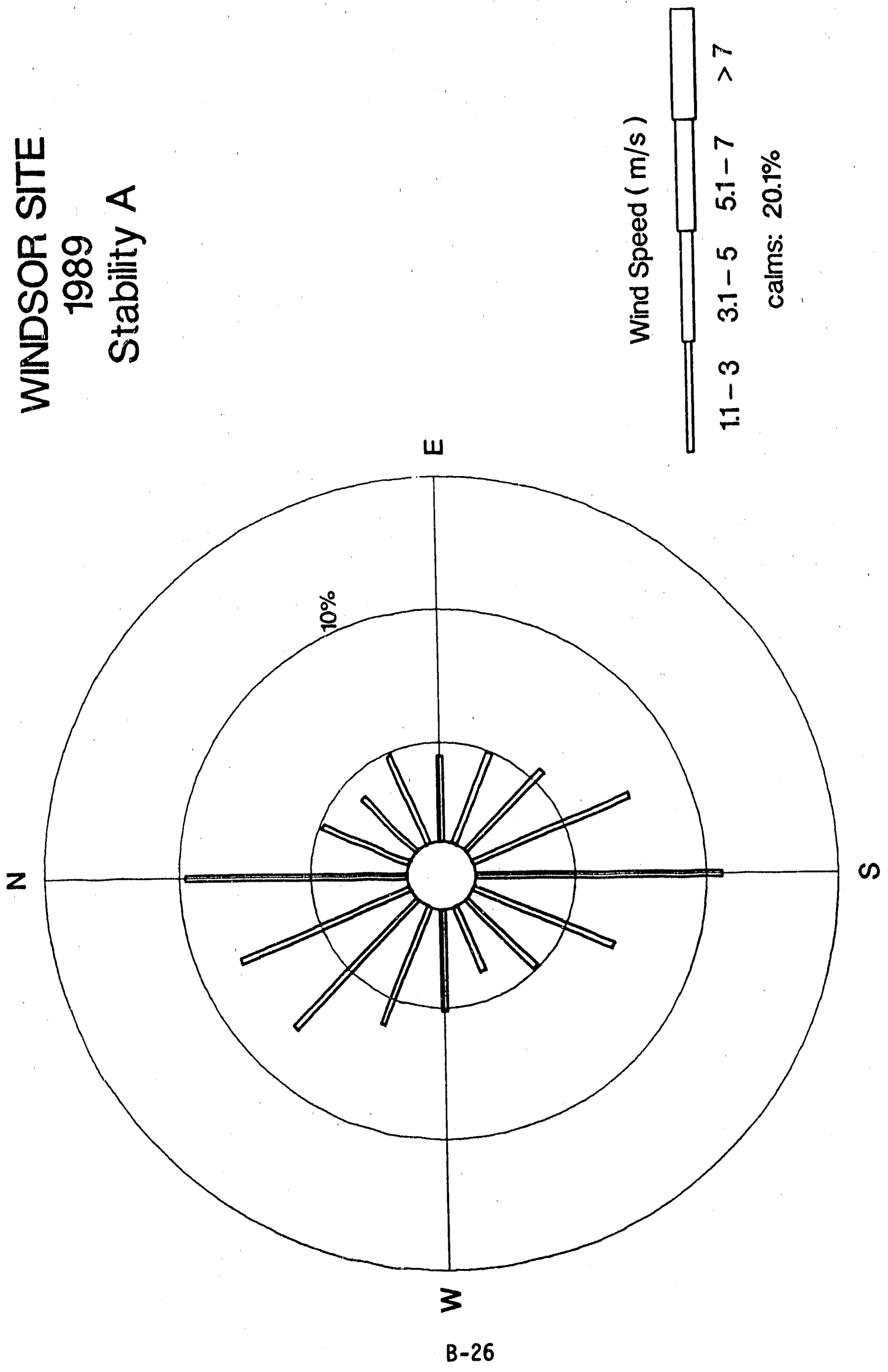




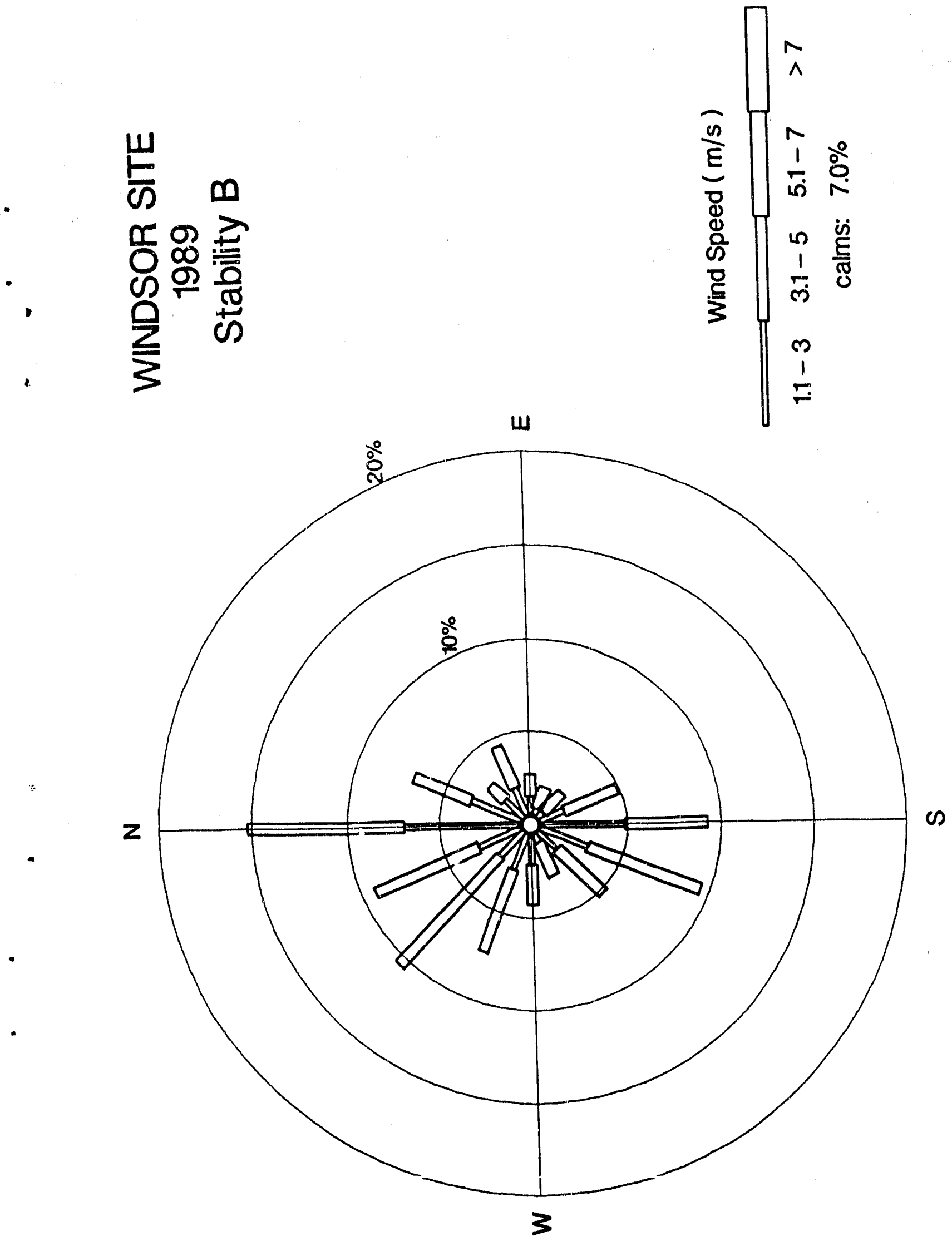




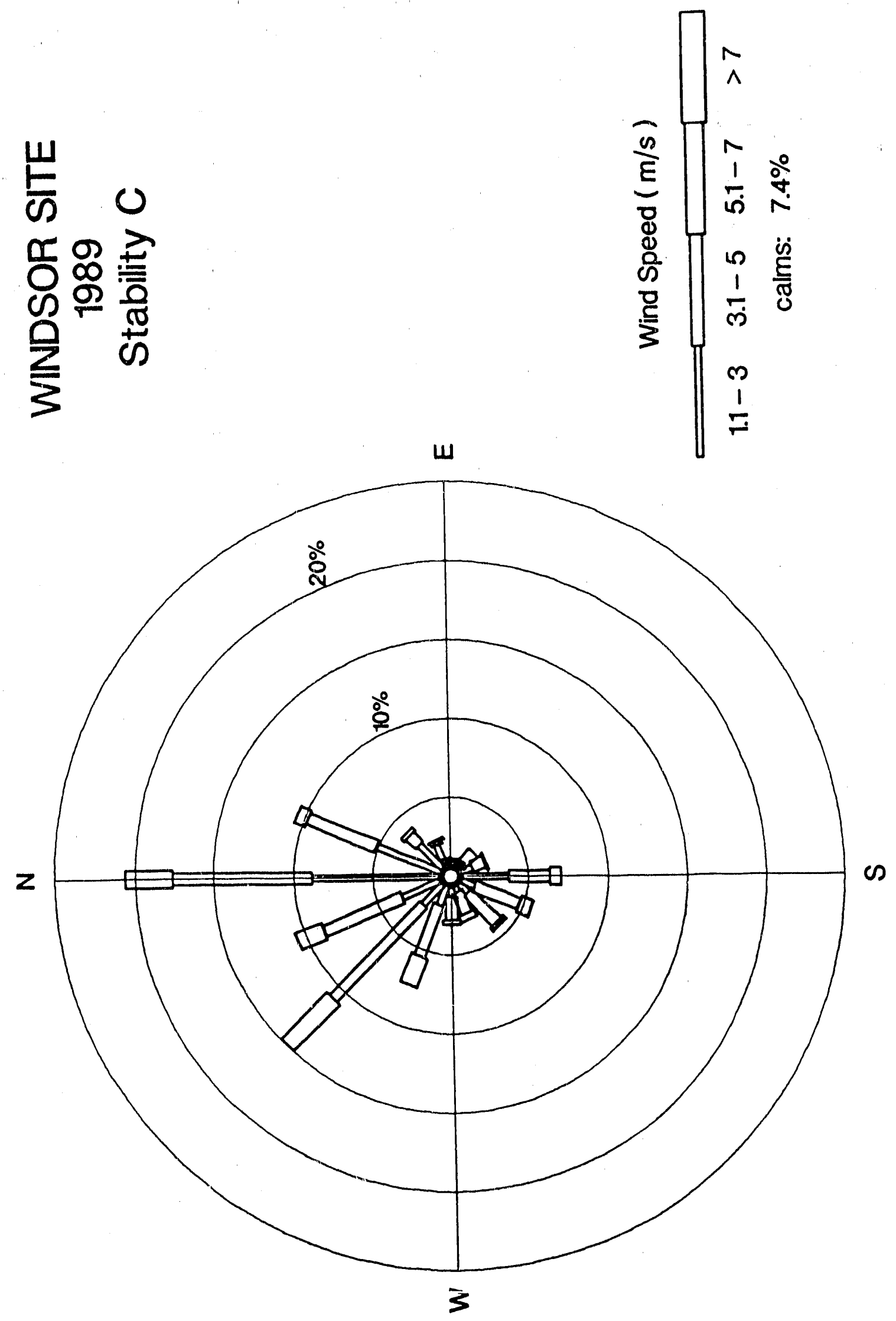




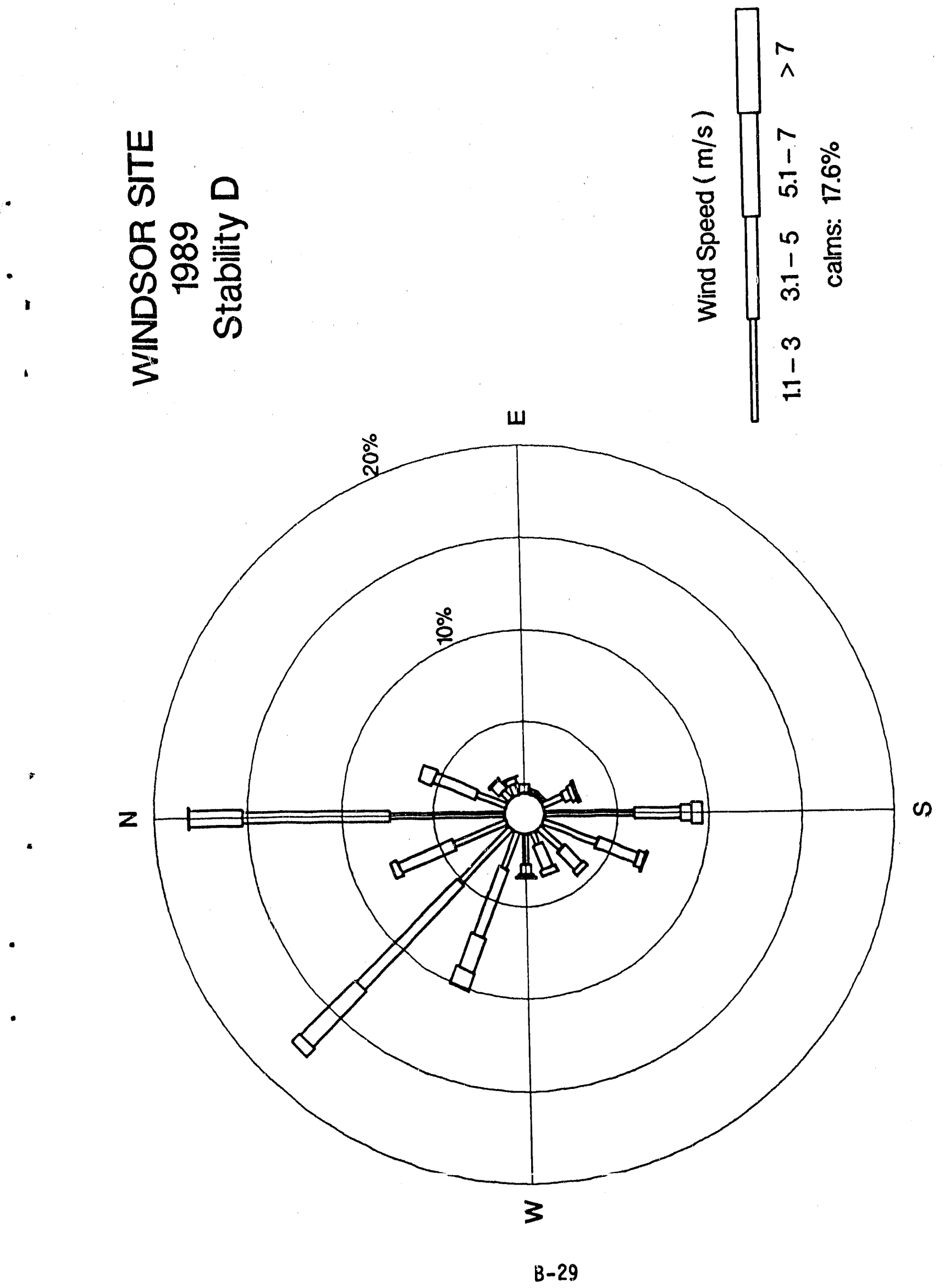



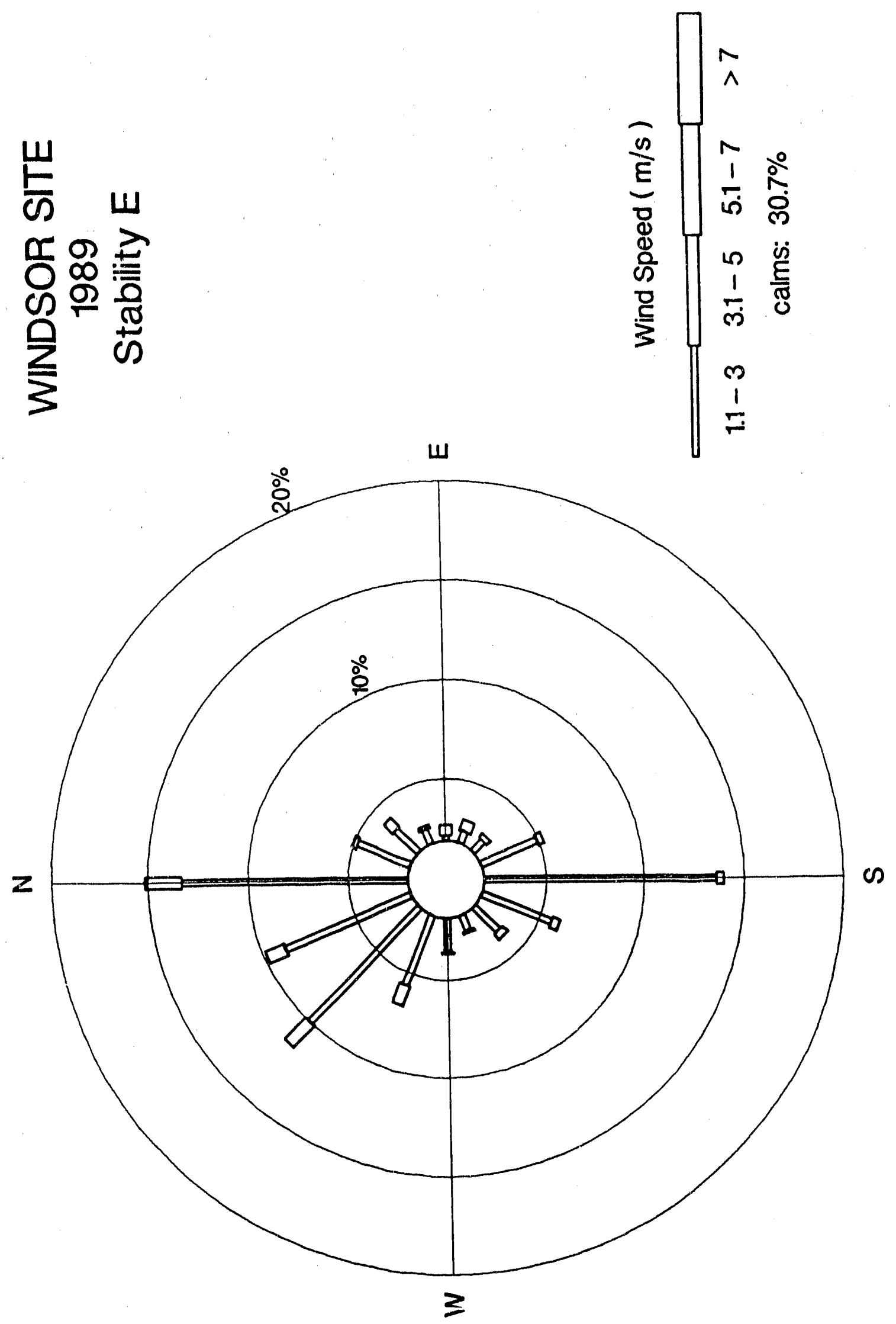


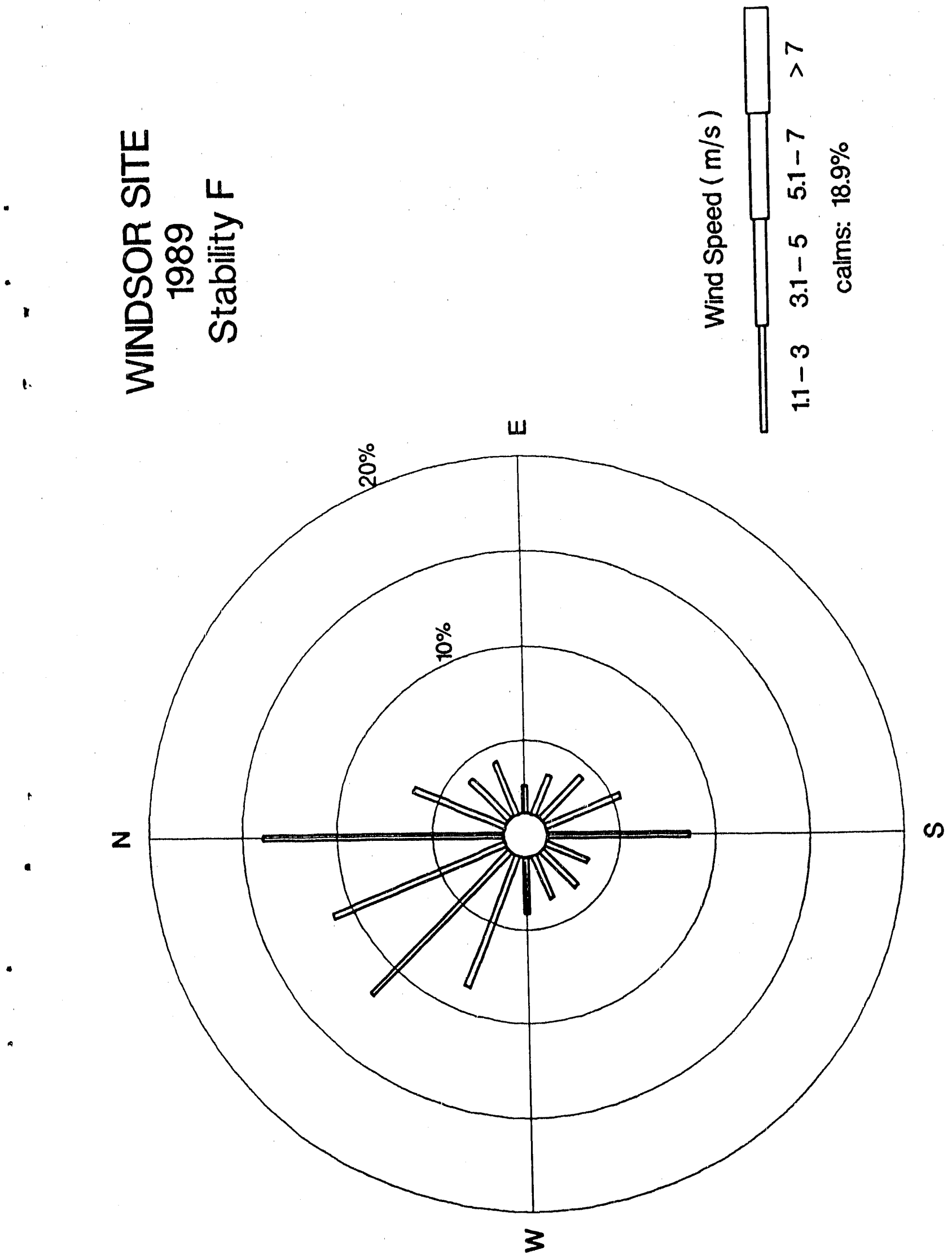



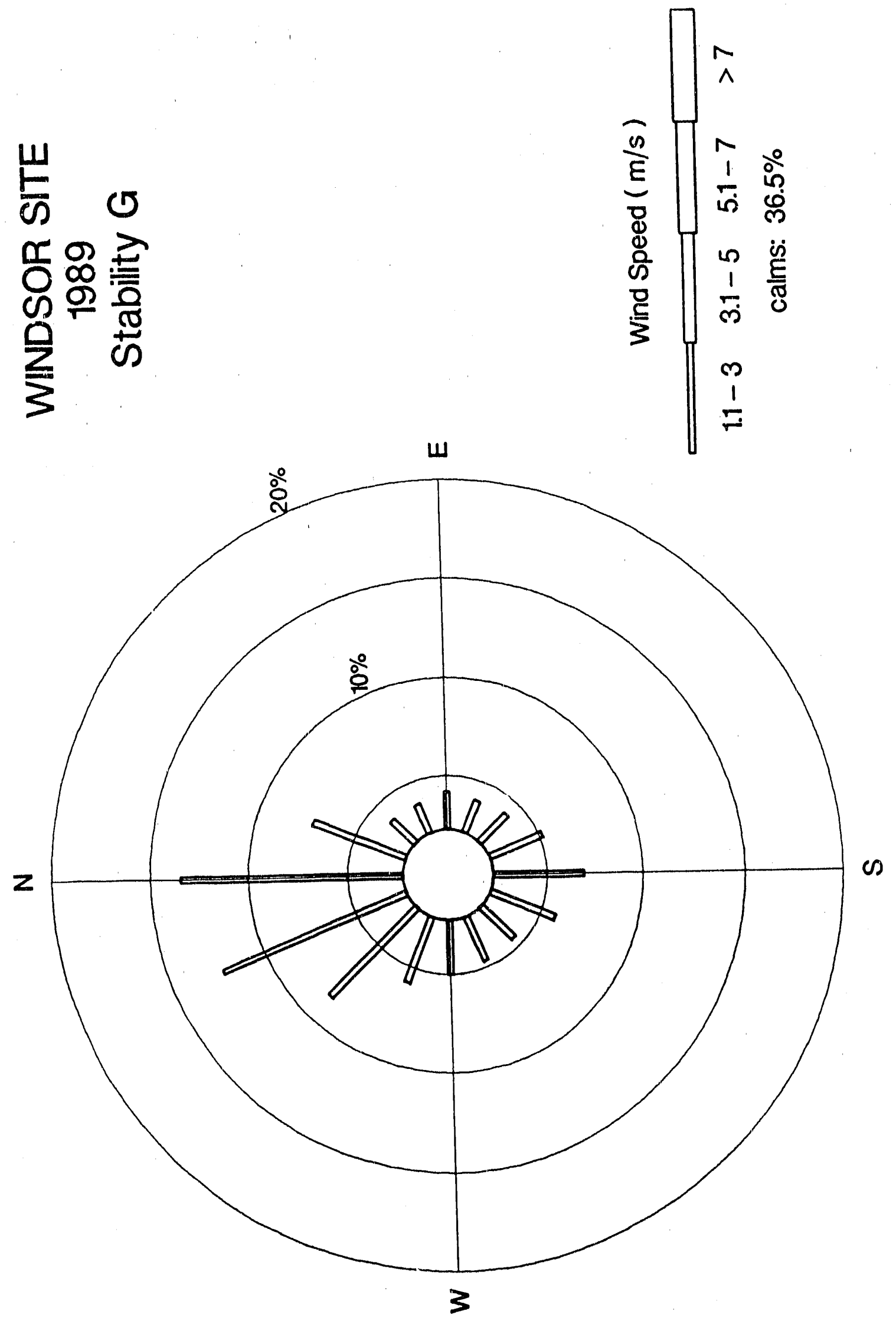


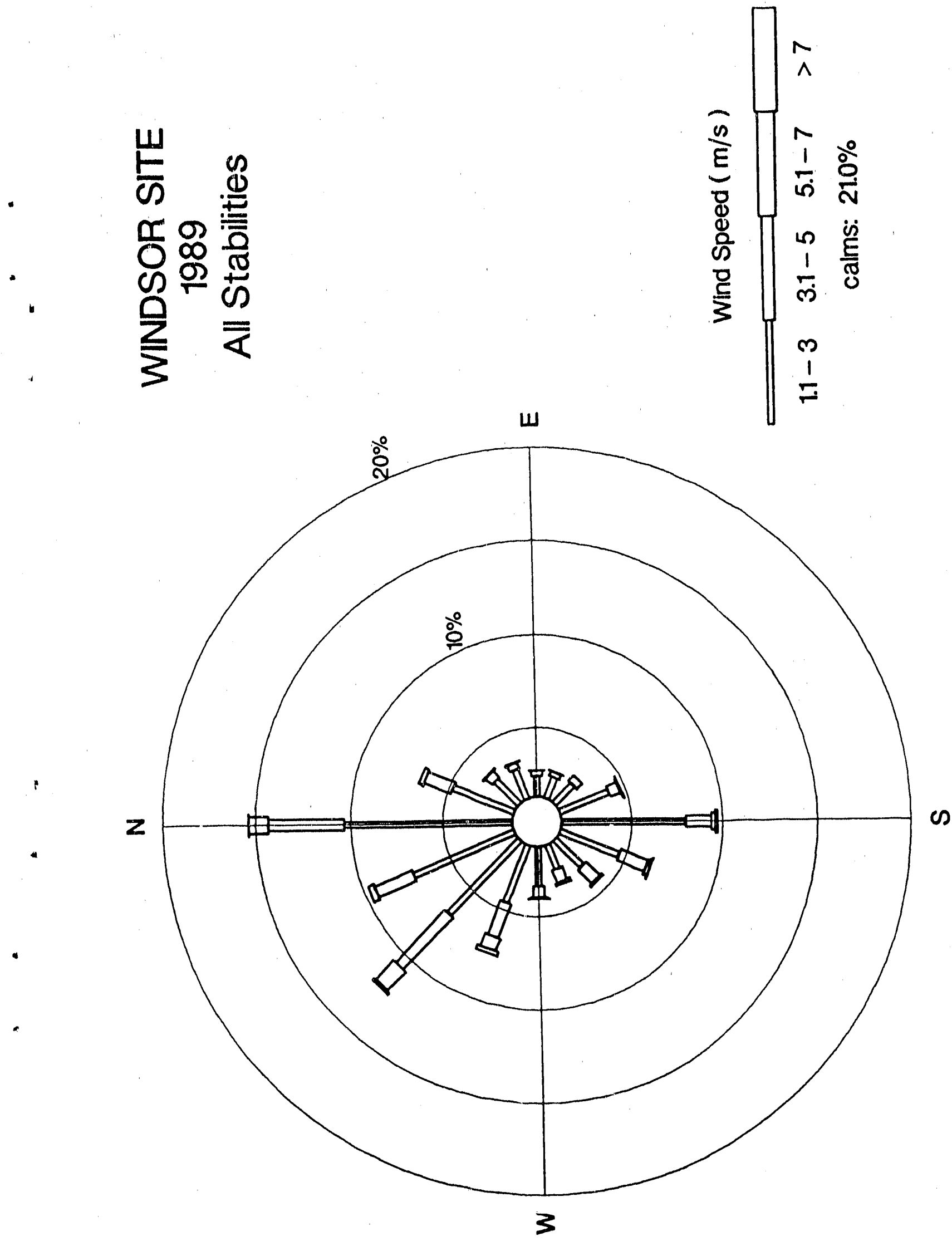




\section{APPENDIX C \\ SOURCE LISTINGS}




\section{HOURK}

This program reads the $15-\mathrm{min}$ average data and produces a listing of hourly averages.

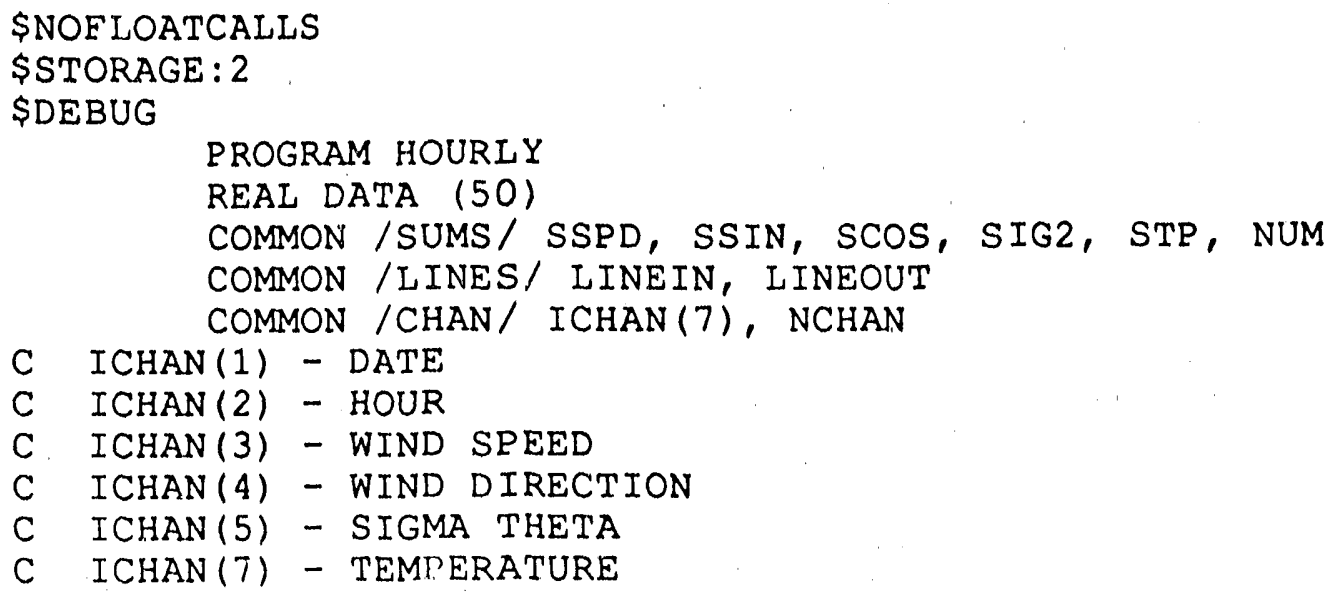

ENDIF

IF (NOWDA .NE. DATA(1) .OR.

1 NOWHR .NE. DATA(2)) THEN

CALL COMPWRT (NOWDA, NOWHR)

CALL CLEAR

ENDIF

NOWDA $=$ DATA $($ ICHAN (1))

NOWHR = DATA $($ ICHAN (2))

$W S=\operatorname{DATA}(I \operatorname{IHAN}(3))$ 
C USE PREVIOUS WD AND SIGMA THETA WHEN WS IS CALM

IF (WS . GE. 0.5 ) THEN

WD $=$ DATA $(\operatorname{ICHAN}(4))$

ENDIF

$S I=$ DATA (ICHAN (5))

$T P=\operatorname{DATA}(\operatorname{ICHAN}(7))$

IF ((WS .EQ. 99.9$)$.OR.

1 (WD .EQ. 999.) .OR.

2 (SI .EQ. 999.9) .OR.

4 (TP.EQ. 99.9)) THEN

ENDIE

GO'TO 8

IF ((WS .LT. 0) .OR. (WS .GT. 30) .OR.

(WD.IT. O) .OR. (WD.GT. 360) .OR .

(SI.LT. 0) .OR. (SI.GT. 360) .OR.

(TP .LT.-30) .OR, (TP.GT. 50)) THEN

WRITE $(*, *)$ 'DATA OUT OF RANGE', WS, WD, SI, TP

WRITE $(*, *)$ 'LINE $=1$, LINEIN

WRITE $(6, *)$ 'DATA OUT OF RANGE', WS, WD, SI, TR

WRITE $(6, *)$ 'LINE $=$ ', LINEIN

STOP

ENDIF

CALL ADDTOSUM (WS, WD, SI, TP)

8 CONTINUE

CALI READONE (ISTAT, DATA)

IF (ISTAT . LT. O) THEN

CALL COMPWRT (NOWDA, NOWHR)

GOTO 3

ENDIF

GOTO 5

END

\$PAGE

SUBROUTINE RDINIT

COMMONS /CHAN/ ICHAN (7), NCHAN

COMMIJN /ZEES/ ZO, Z

OPEN (3, FILE='HOURK. INI', STATUS='OLD')

NCHAN $=0$

$\operatorname{READ}(3, *)$

$\operatorname{READ}(3, *)$ ZO

WRITE $\left(6,{ }^{*}\right)$ 'Surface roughness $(\mathrm{cm})=1, \mathrm{zO}$

$\operatorname{READ}(3, \star) \mathrm{Z}$

WRITE $(6, *)$ 'Height of instruments $(\mathrm{cm})=1,2$

DO $5 I=1,7$

$\operatorname{READ}(3, *, \mathrm{END}=6)$ ICHAN (I)

NCHAN $=\operatorname{MAX}($ NCHAN, ICHAN $(I))$ 


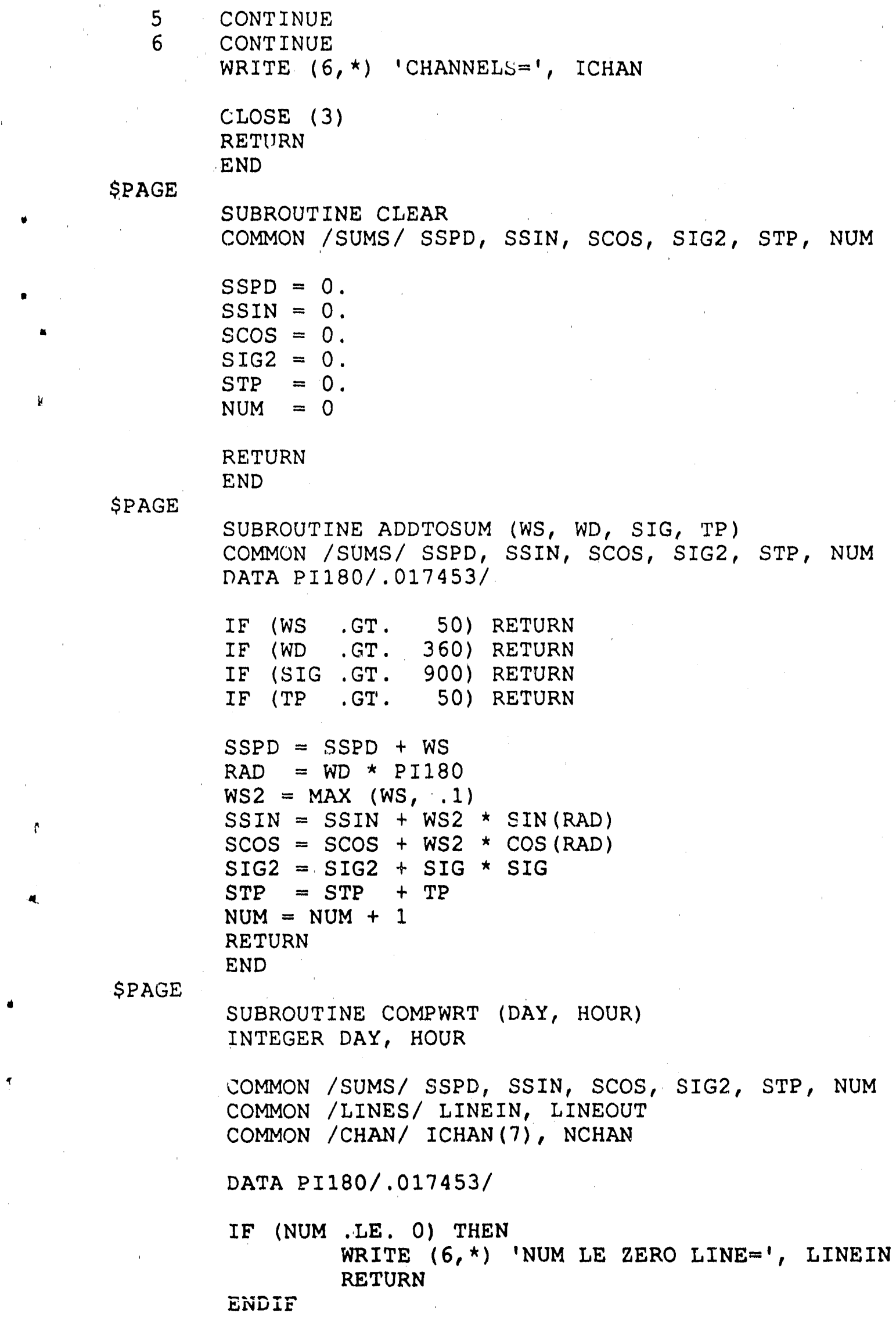




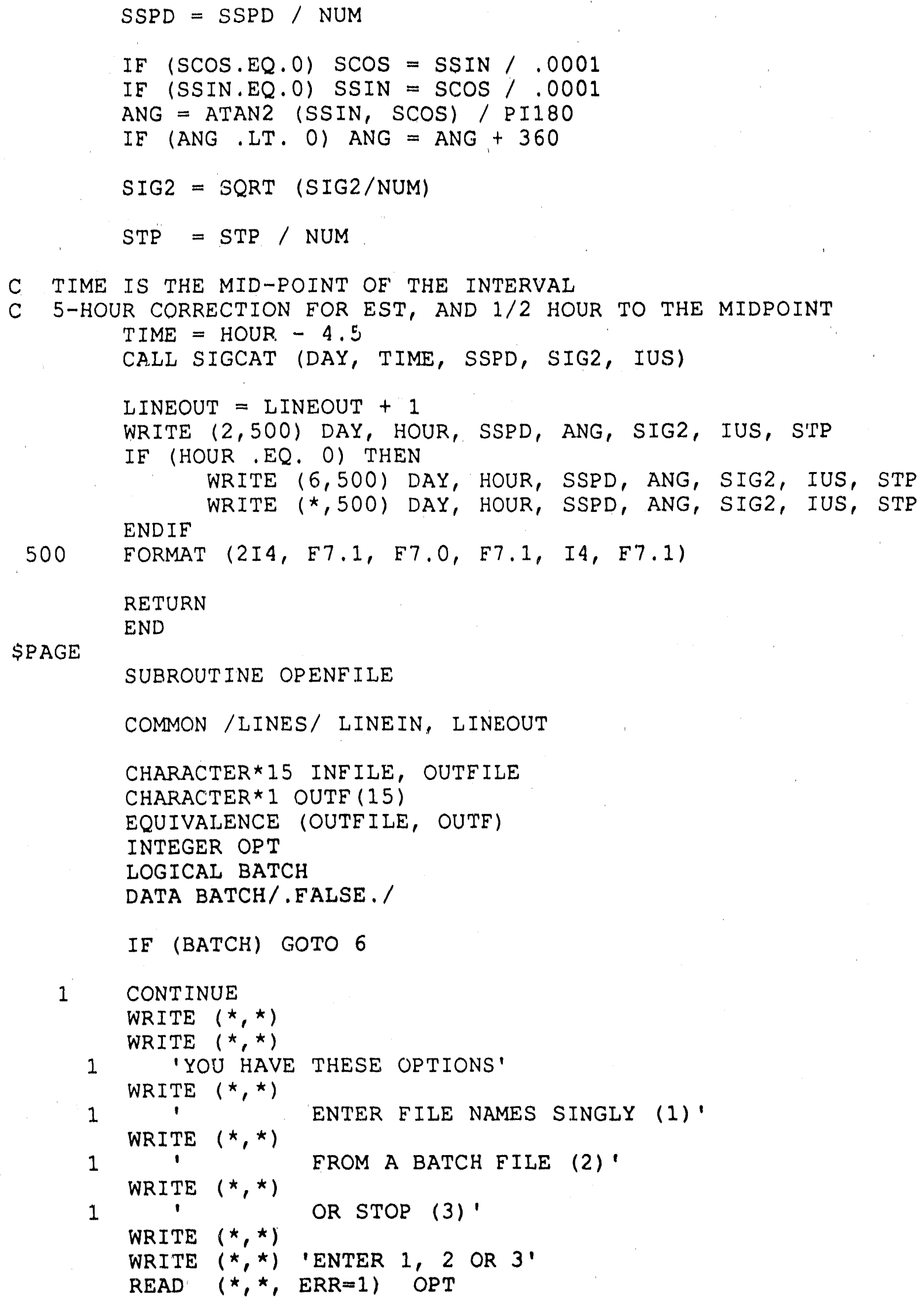




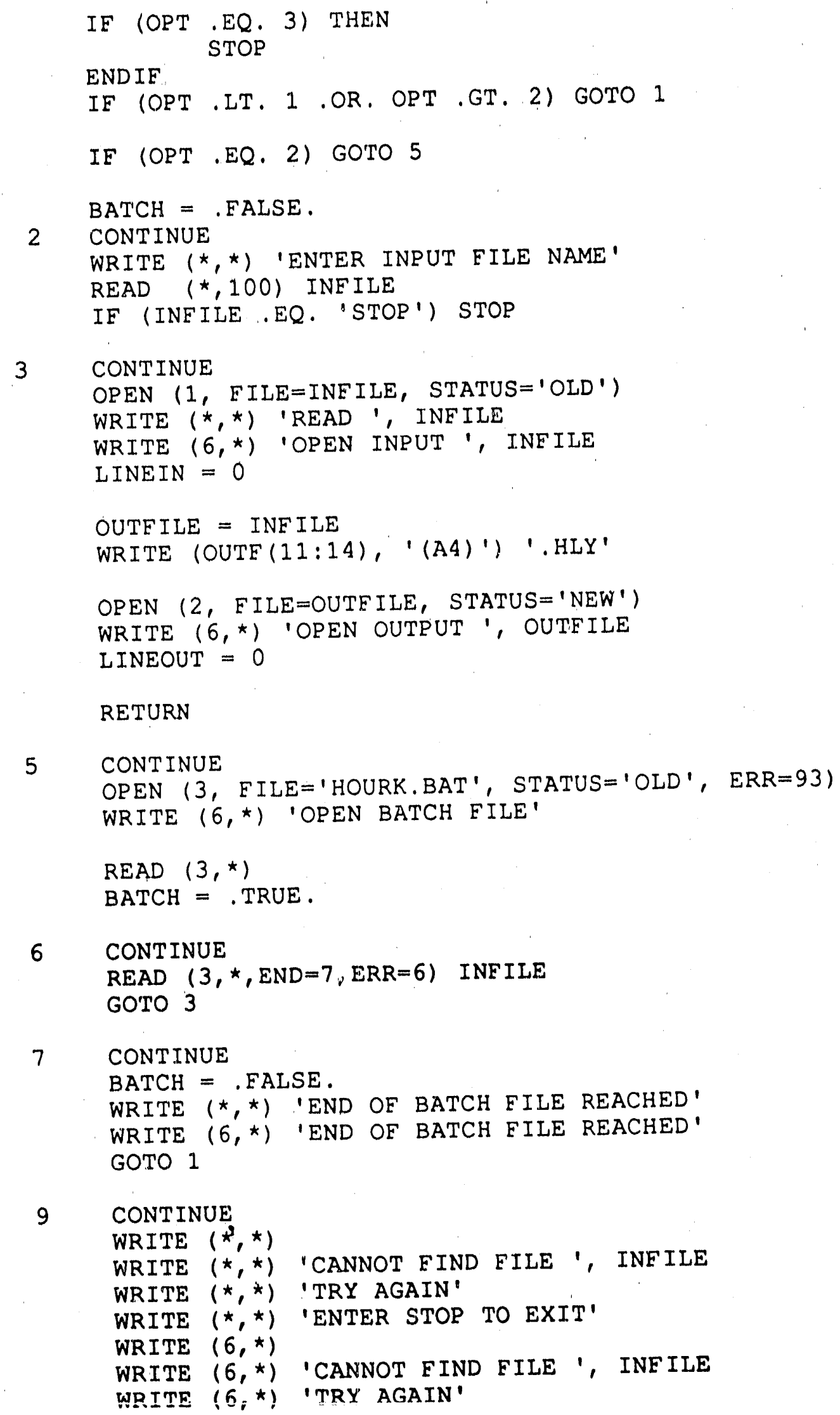

9 CONTINUE 


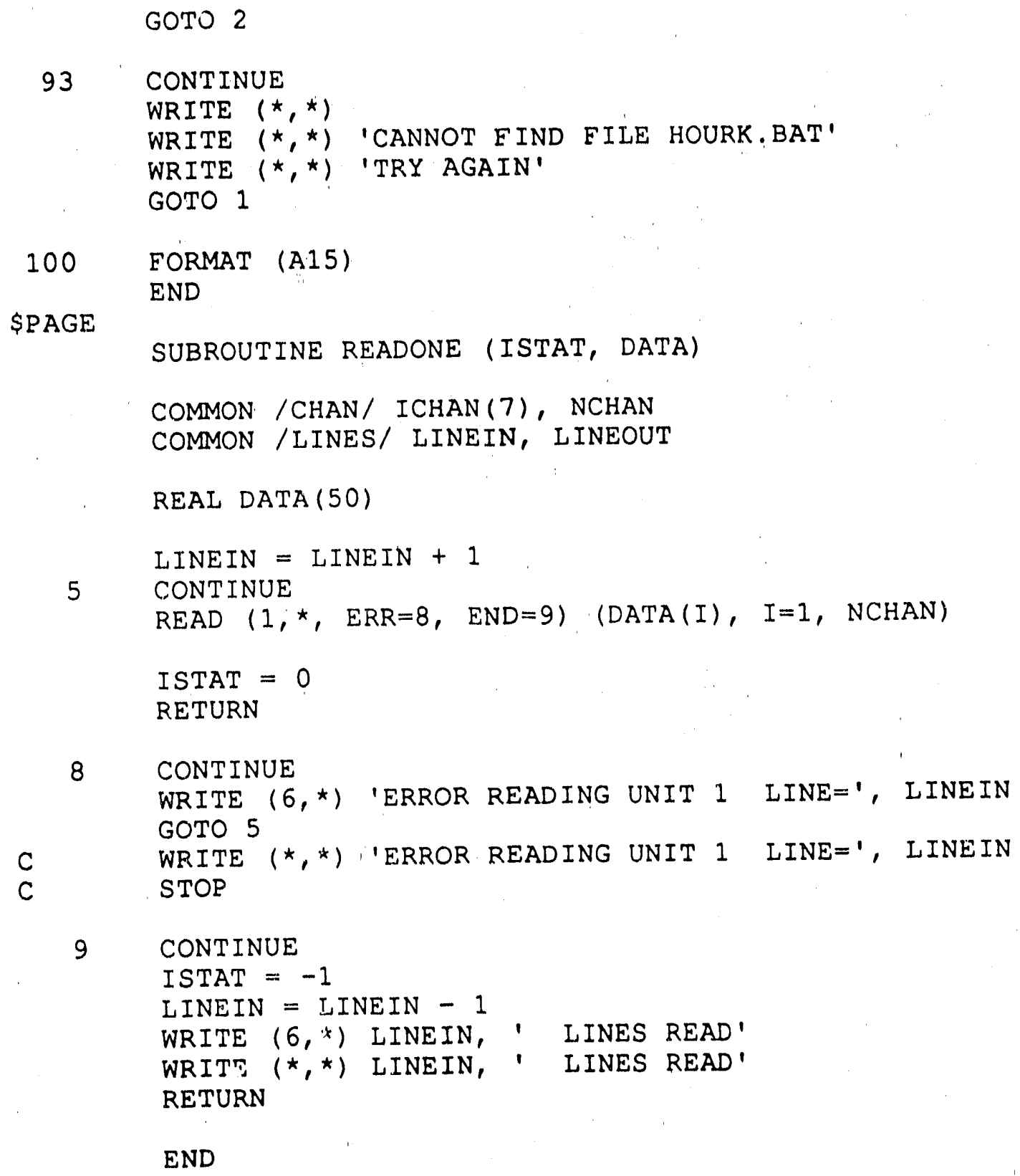

END 


\section{SIGCATK}

\$NOFLOATCALLS

\$STORAGE : 2

SDE.BUG

SLBROUTINE SIGCAT (JUL, TIME, WS, SIGTHT, ISTB)

C This subroutine converts a given value of sigma theta ("SIGTHT")

C to a Pasquill stability category.

C This conversion was first quoted in Nuclear Safety $17(1): 72$

C (F.A. Gifford, 1976) and can be found in the Handbook on

C Atmospheric Diffusion, S.R. Hanna, G.A. Briggs, R.P. Hosker, Jr.,

C U.S. DOE (1982), NTIS\# DOE/TIC-11223 (DE82002045), Table 4.3 .

COMMON /ZEES/ 20, 2

$\operatorname{REAL} \operatorname{RISET}(2,365), \operatorname{LOWLIM}(7), \operatorname{EXP}(7)$

LOGICAL IHERE

DATA HERE/.FALSE./

DATA LOWLIM/22.5, 17.5, 12.5, 7.5, 3.8, 2.1,

$0.43 /$

DATA EXP $1-0.06,-0.15,-0.17,-0.23,-0.38,-0.43$, -

TE (.NOT.HERE) THEN

OPEN (4, FILE='SUNRISET.DAT', STATUS='OLD')

$\operatorname{READ}(4, *)$

DO $3 I=1,365$

$\operatorname{READ}\left(c_{1}, \star\right) J, \operatorname{RISET}(1, I), \operatorname{RISET}(2, I)$

3

CONTINUE

CLOSE (4)

HERE $=$.TRUE.

WRITE $(6, *) ' Z 0, Z=1,20, z$

DO $35 I=1,7$

FACTOK $=(20 / 15) \star \star(0.2) \star(2 / 10) \star \star \operatorname{EXP}(I)$

35

LOWLIM $(I)=$ LOWLIM $(I) \star$ EACTOR

CONTINUE

ENDIF

WRITE $\left(6,{ }^{*}\right)$ 'LOW IIMITS', LOWLIM

C IF THE WIND SPEED IS GREATER THAN $6 \mathrm{~m} / \mathrm{s}$ STABILITY IS NEUTRAL IF (WS . GT. 6) THEN

ISTB $=4$

ENDIF

COTO 5

C CONVERT SIGMA THETA TO A DAYTIME STABILITY CLASS

$I S T B=0$ 
4 CONTINUE

$I S T B=I S T B+1$

IF (SIGTHT . LT. LOWLIM(ISTB)) GOTO 4

C MAKE ROOM FOR THE NIGHTIME NEUTRAL CASE (ISTB $=5$ )

IF (ISTB .GE. 5) THEN

ENDIF

ISTB $=$ ISTB +1

5 CONTINUE

C TEST FOR NIGHTIME CONDITIONS

C NIGHTIME IS CONSIDERED TO BE FROM 1 HOUR BEFORE SUNSET

C TO 1 HOUR AFTER SUNRISE.

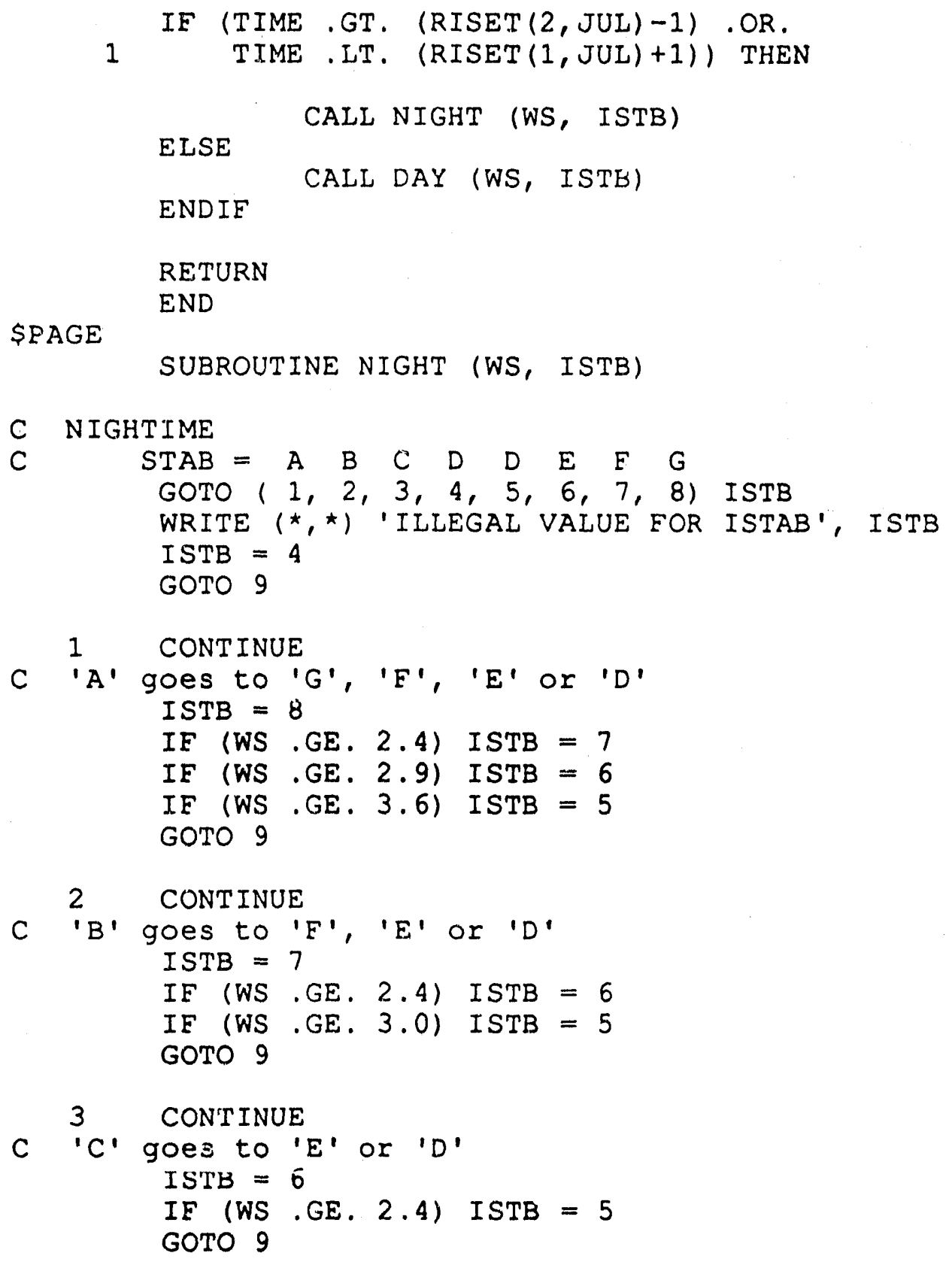




$$
\begin{aligned}
& \begin{array}{l}
4 \text { CONTINUE } \\
C \quad \text { 'D' at night } \\
\text { ISTB }=5 \\
\text { GOTO } 9
\end{array} \\
& \begin{array}{c}
\text { 5. CONTINUE } \\
\text { C.D' at night } \\
\text { GOTO } 9
\end{array} \\
& \begin{array}{ll}
6 \quad \text { CONTINUE } \\
\quad \text { ISTB }=6 \\
\\
\quad \text { IF (WS .GE, 5.0) ISTB }=5 \\
\text { GOTO } 9
\end{array} \\
& \begin{aligned}
7 . & \text { CONTINUE } \\
& \\
& \text { ISTB }=7 \\
& \text { IF (WS.GE. 3.0) } \text { ISTB }=6 \\
& \text { IE (WS.GE. 5.0) } \text { ISTB }=5
\end{aligned} \\
& \begin{array}{ll}
8 . & \text { CONTINUE } \\
\text { I }^{\prime} & \\
& \text { ISTB }=8 \\
& \text { IE (WS .GE. 3.0) } \text { ISTB }=6 \\
& \text { IF (WS.GE. 5.0) } \text { ISTB }=5
\end{array} \\
& \begin{array}{cl}
9 & \text { RETURN } \\
\text { END } \\
\text { \$PAGE } & \\
& \text { SUBROUT INE DAY (WS, ISTB) }
\end{array}
\end{aligned}
$$

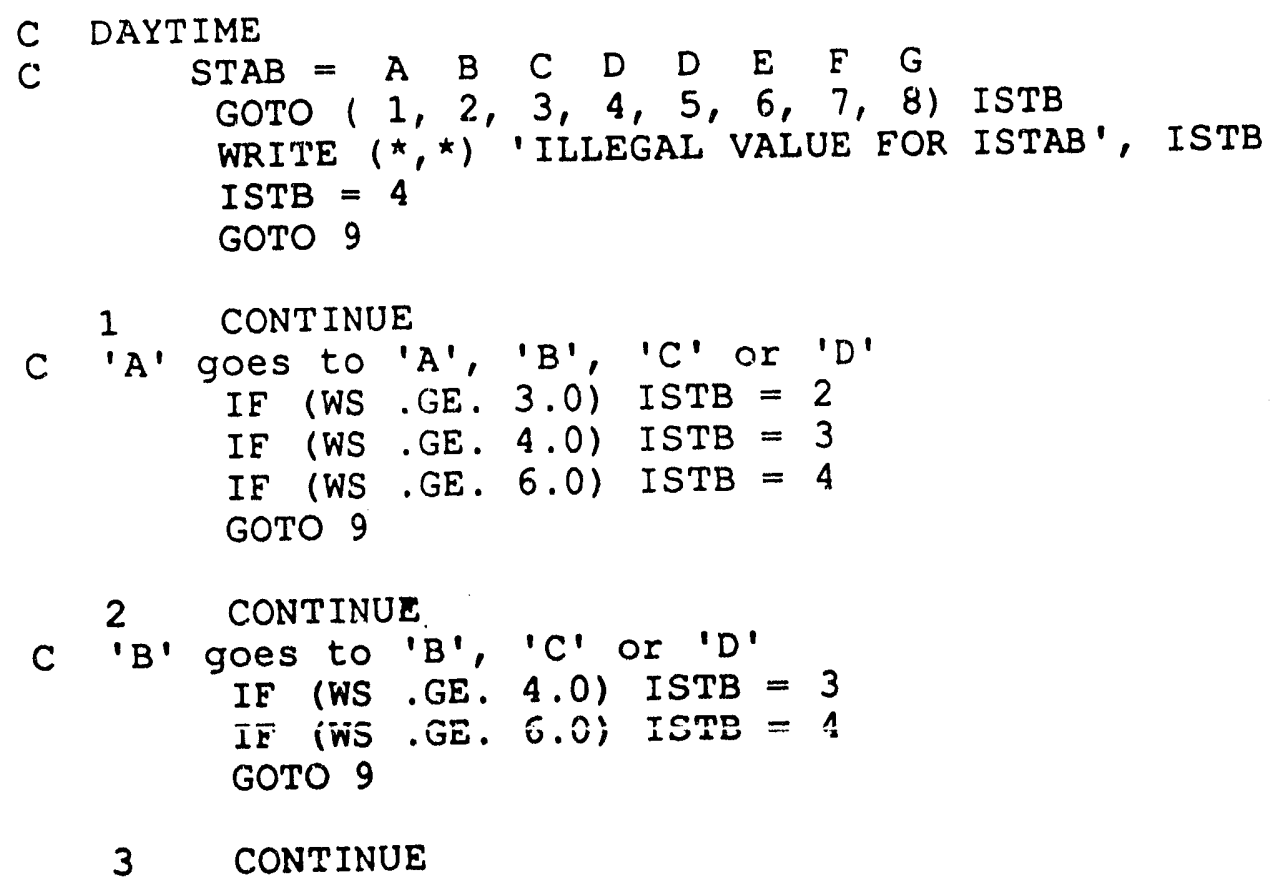




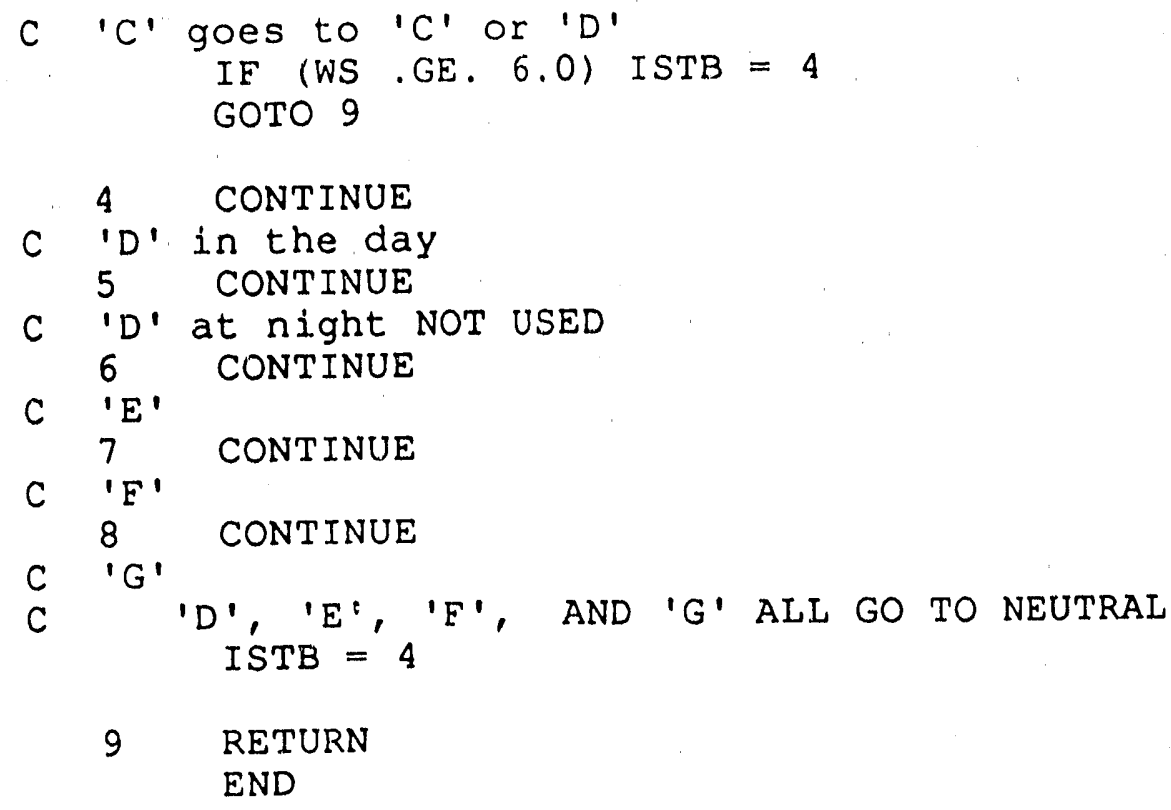



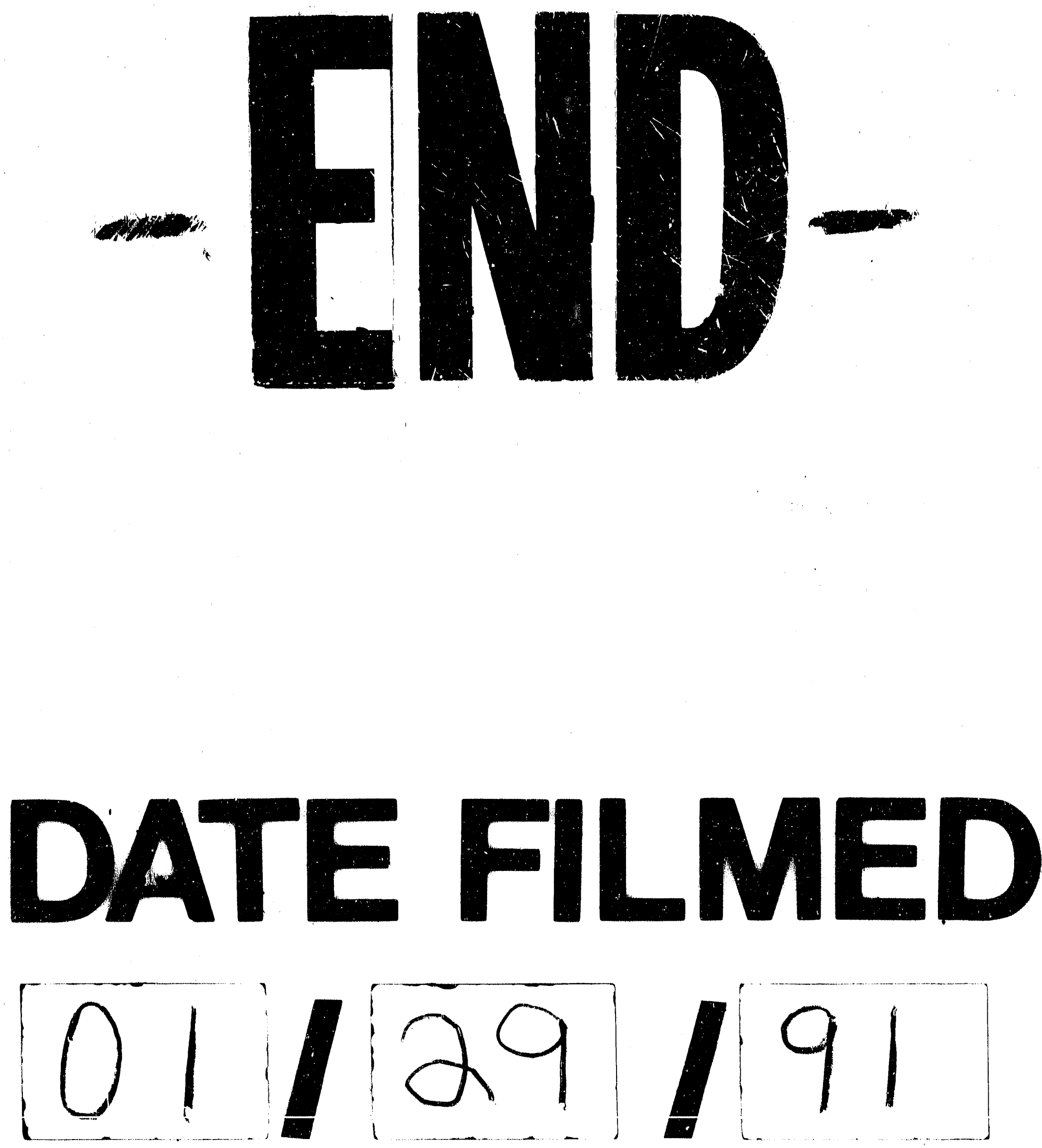
